Ergod. Th. \& Dynam. Sys. (1984), 4, 323-351

Printed in Great Britain

\title{
Weak disjointness and the equicontinuous structure relation
}

\author{
JOE AUSLANDER, DOUG MCMAHON, JAAP VAN DER WOUDE \\ AND TA SUN WU
}

Department of Mathematics, University of Maryland, College Park, MD 20742, USA; Department of Mathematics, Arizona State University, Tempe, AZ 85281, USA;

Department of Mathematics and Statistics, Case Western Reserve University, Cleveland, $\mathrm{OH} 44106$, USA;

Subfaculteit Wiskunde, Vrije Universiteit, Amsterdam, The Netherlands

(Received 23 March 1983 and revised 14 February 1984)

Abstract. We discuss weak disjointness of homomorphisms of minimal transformation groups and use the techniques involved to deepen our knowledge of the equicontinuous structure relation.

\section{Introduction and notation}

A topological transformation group (ttg) is a triple $\mathscr{X}=\langle T, X, \pi\rangle$, where $T$ is a $T_{2}$ topological group, $X$ is a compact $\mathrm{T}_{2},\left(\mathrm{CT}_{2}\right)$, space and $\pi: T \times X \rightarrow X$ is a continuous map such that $\pi(e, x)=x$ and $\pi(s, \pi(t, x))=\pi(s t, x)$. I.e. $T$ acts as a continuous group of homeomorphisms on $X$. We shall fix $T$ and suppress the action symbol.

Let $\mathscr{Z}$ be a ttg, $x \in X$, then $T x(\overline{T x})$ denotes the orbit (-closure) of $x$ in $X$ and a subset $A \subseteq X$ is called invariant iff $T a \subseteq A$ for every $a \in A$. A ttg $\mathscr{X}$ is called minimal iff $X$ contains no proper closed invariant subsets, $\mathscr{X}$ is called ergodic iff every invariant open subset of $X$ is dense. An example of an ergodic ttg is a point-transitive $\mathrm{ttg}$, which is a ttg with a dense orbit. A ttg is minimal iff every orbit is dense.

For (our fixed) $T$ there exists a universal point-transitive $\operatorname{ttg} \mathscr{S}_{T}$, such that $T$ can densely and equivariantly be embedded in $S_{T}$. The multiplication on $T$ can be extended to a multiplication on $S_{T}$, then $S_{T}$ is a closed semigroup with continuous right translations. The universal minimal $\operatorname{ttg} \mathfrak{M}=\langle T, M\rangle$ for $T$ is isomorphic to every minimal left ideal in $S_{T}$ and so $M$ is a closed semigroup with continuous right translations. Hence the collection $J:=J(M)$ of idempotents in $M$ is non-empty. Moreover, $\{v M \mid v \in J\}$ is a partition of $M$ and every $v M$ is a group with unit element $v$.

The sets $S_{T}$ and $M$ act on $X$ as semigroups and $\overline{T x}=S_{T} x$, while for a minimal $\operatorname{ttg} \mathscr{X}$ we have $\overline{T x}=M x$ for every $x \in X$. Let $x$ be an almost periodic point in $X$, i.e. $\overline{T x}$ is minimal, then denote the non-empty set $\{v \in J \mid v x=x\}$ by $J_{x}$. Note, that for a $\operatorname{ttg} \mathscr{X}, J X$ is the collection of almost periodic points in $X$.

Proximality is another dynamical concept that can be described by the action of $S_{T}$. Two points $x_{1}$ and $x_{2}$ are called proximal iff

$$
\overline{T\left(x_{1}, x_{2}\right)} \cap \Delta_{X} \neq \varnothing \text {. }
$$


Let $U_{X}$ be the unique uniform structure of $X$, then

$$
P=\bigcap\left\{T \alpha \mid \alpha \in \mathscr{U}_{X}\right\}
$$

is the collection of proximal pairs in $X$, the proximal relation; if $P=X \times X$ then $\mathscr{Z}$ is called proximal. It turns out that $x_{1}$ and $x_{2}$ are proximal in $\mathscr{X}$ iff $v x_{1}=v x_{2}$ for some idempotent $v \in S_{T}$ iff there is a minimal left ideal $I$ in $S_{T}$ such that $p x_{1}=p x_{2}$ for every $p \in I$.

Let $2^{X}$ be the collection of non-empty closed subsets of $X$ endowed with the Vietoris topology [13]. Note that a base for the Vietoris topology on $2^{X}$ is formed by the sets

$$
\left\langle U_{1}, \ldots, U_{n}\right\rangle:=\left\{A \in 2^{X} \mid A \subseteq \bigcup_{i=1}^{n} U_{i} \text { and } A \cap U_{i} \neq \varnothing \text { for every } i\right\},
$$

where $U_{i}$ is open in $X$. Then $2^{\mathscr{L}}:=\left\langle T, 2^{X}, \tilde{\pi}\right\rangle$ defined by $\tilde{\pi}(t, D):=\pi[\{t\} \times D]$ is a ttg again, and $S_{T}$ acts on $2^{X}$ too. To avoid ambiguity we denote the action of $S_{T}$ on $2^{X}$ by the circle operation as follows. Let $p \in S_{T}$, then for $D \in 2^{X}$ define $p \circ D:=\lim _{2} \times t_{i} D$ for any net $\left\{t_{i}\right\}_{i}$ in $T$ with $t_{i} \rightarrow p$. Moreover

$$
p \circ D=\left\{x \in X \mid \text { there are } d_{i} \in D \text { with } x=\lim t_{i} d_{i}\right\},
$$

for any net $t_{i} \rightarrow p$ in $T$. If $F \subseteq S_{T}, D \in 2^{X}$ then we define

$$
F \circ D:=\bigcup\{f \circ D \mid f \in F\} \text {. }
$$

A homomorphism of ttgs $\phi: \mathscr{X} \rightarrow \mathscr{Y}$ is a continuous map $\phi: X \rightarrow Y$ of the phase spaces of the ttgs such that $\phi(t x)=t \phi(x)$ for all $t \in T, x \in X$. Define

$$
R_{\phi}:=\left\{\left(x_{1}, x_{2}\right) \mid \phi\left(x_{1}\right)=\phi\left(x_{2}\right)\right\},
$$

then $Y \cong X / R_{\phi}$. The map $\phi$ is called proximal iff $R_{\phi} \subseteq P$ iff $P_{\phi}:=$ $\bigcap\left\{T \alpha \cap R_{\phi} \mid \alpha \in \mathscr{U}_{X}\right\}=R_{\phi}$.

Let $\phi: \mathscr{Z} \rightarrow \mathscr{Z}$ and $\psi: \mathscr{Y} \rightarrow \mathscr{Z}$ be surjective homomorphisms of ttgs, then $\phi$ and $\psi$ are called disjoint iff $R_{\phi \psi}:=\{(x, y) \mid \phi(x)=\psi(y)\}$ is minimal, notation $\phi \perp \psi ; \phi$ and $\psi$ are called weakly disjoint iff $R_{\phi \psi}$ is ergodic, notation $\phi-\psi$.

In $\$ 2$ we shall relate weak disjointness of homomorphisms of ttgs to that of their maximally equicontinuous factors. A homomorphism of ttgs is called equicontinuous or almost periodic iff for every $\alpha \in \mathcal{U}_{X}$ there is a $\beta \in \mathcal{U}_{X}$ such that $T \alpha \cap R_{\phi} \subseteq \beta$. Let

$$
Q_{\phi}:=\bigcap\left\{\overline{T \alpha \cap R_{\phi}} \mid \alpha \in U_{x}\right\}
$$

be the regionally proximal relation and define the equicontinuous structure relation $E_{\phi}$ to be the smallest closed invariant equivalence relation that contains $Q_{\phi}$. Then $\phi: \mathscr{X} \rightarrow \mathscr{Y}$ is almost periodic iff $Q_{\phi}=\Delta_{X}$. If $\phi: \mathscr{X} \rightarrow \mathscr{Y}$ is a homomorphism of ttgs, then $\theta: \mathscr{X} / E_{\phi} \rightarrow \mathscr{Y}$ is the maximal equicontinuous factor of $\phi$ (also called the maximal (uniformly) almost periodic factor of $\phi$ ).

Before we can discuss the material in $\S 2$ we need to collect some results concerning the equicontinuous structure relation of certain types of homomorphisms of minimal ttgs, which will be done in $\S 1$. Most of the results in $\S 1$ are known, but the results in $1.9 \mathrm{~d}$ and $1.16 \mathrm{~b}$ seem to be new.

In $\S 3$ we refine our knowledge about the regionally proximal relation being an equivalence relation. We prove that in several cases (RIC, Bc, RIM see $\S 1$ for 
definitions) we have

$$
E_{\phi}=Q_{\phi}=\cap\left\{\text { int }_{R_{\phi}} \overline{T \alpha \cap R_{\phi}} \mid \alpha \in U_{X}\right\} \quad\left(=Q_{\phi}^{\#}\right) .
$$

Note that it was already known that $E_{\phi}=Q_{\phi}$ for open $\mathrm{Bc}$ extensions [2], Bc extensions [15] and open RIM extensions [16].

In $\$ 4$ we return to the transitivity of the regionally proximal relation and translate some of the '\#-ideas' as revealed in $\S 3$ to the idea of regional proximality of second order.

A more detailed discussion about ttgs may be found in [3] and, with more notational resemblance, [10] and [15]; for a function-algebraic approach see [5].

We would like to thank $\mathbf{J}$. de Vries for his many valuable suggestions, and $T$. $S$. McWoulander for his communications.

\section{Some generalities}

Let $X$ be a $\mathrm{CT}_{2}$ space and let $\mathfrak{M}(X)$ be the collection of regular Borel probability measures on $X$ provided with the weak star topology; i.e. a net $\left\{\mu_{i}\right\}_{i}$ in $\mathfrak{M}(X)$ converges to $\mu \in \mathfrak{M}(X)$ iff $\int f d \mu_{i}$ converges to $\int f d \mu$ for all real valued continuous functions $f$ on $X$. Then $\mathfrak{M}(X)$ is a $\mathrm{CT}_{2}$ space in which $X$ is embedded by the mapping $x \mapsto \delta_{x}$, where $\delta_{x}$ is the dirac measure at $x$. If $\phi: \mathscr{X} \rightarrow \mathscr{Y}$ is a continuous map between $\mathrm{CT}_{2}$ spaces, then $\phi$ induces a continuous map $\mathfrak{M}(\phi): \mathfrak{M}(X) \rightarrow \mathfrak{M}(Y)$ which extends $\phi$. Note, that $\mathfrak{W}(\phi)$ is surjective (injective) (homeomorphic) iff $\phi$ is.

Let $\mathscr{Z}$ be a $\operatorname{ttg}$ for $T$. For $t \in T$ and $\mu \in \mathfrak{M}(X)$ define $t \mu \in \mathfrak{M}(X)$ by $t \mu(A)=$ $\mu\left(t^{-1} A\right)$; or, what is the same, $\int f d(t \mu)=\int f t d \mu$, where $f t: X \rightarrow \mathbb{R}$ is defined by $f t(x)=f(t x)$. Also one could say $t \mu:=\mathfrak{M}\left(\pi^{t}\right)(\mu)$, where $\pi^{t}:=x \mapsto t x: X \rightarrow X$. One can show that

$$
(t, \mu) \mapsto t \mu: T \times \mathfrak{M}(X) \rightarrow \mathfrak{M}(X)
$$

is continuous. So $\mathfrak{W}(\mathscr{X})$ is a ttg for $T$. If $\phi: \mathscr{X} \rightarrow \mathscr{Y}$ is a homomorphism of ttgs, then $\mathfrak{M}(\phi): \mathfrak{M}(\mathscr{X}) \rightarrow \mathfrak{M}(\mathscr{Y})$ is a homomorphism of ttgs.

A surjective homomorphism $\phi: \mathscr{X} \rightarrow \mathscr{Y}$ of ttgs is said to have a relatively invariant measure ( $\phi$ has a RIM, $\phi$ is a RIM extension) if there exists a continuous homomorphism $\lambda: \mathscr{Y} \rightarrow \mathfrak{M}(\mathscr{X})$ of ttgs such that

$$
\mathfrak{M}(\phi) \circ \lambda: \mathscr{Y} \rightarrow \mathfrak{M}(\mathscr{Y})
$$

is just the (dirac) embedding. In other words: $\phi$ is a RIM extension iff for every $y \in Y$ there is a $\lambda_{y} \in \mathfrak{M}(X)$ with supp $\lambda_{y} \subseteq \phi^{\leftarrow}(y)$ and the map $y \mapsto \lambda_{y}: \mathscr{Y} \rightarrow \mathfrak{M}(\mathscr{X})$ is a homomorphism of ttgs; this map $\lambda$ is called a section for $\phi$. If $\phi$ is a RIM extension, then. a point $x \in X$ is called a supprim point if for some section $\lambda$ for $\phi$ we have $x \in \operatorname{supp} \lambda_{\phi(x)}$. In particular, $\phi: \mathscr{X} \rightarrow\{\star\}$ has a RIM iff $\mathscr{X}$ has an invariant measure iff $\mathfrak{M}(\mathscr{X})$ has a fixed point. In case $\mathscr{X}$ is minimal it follows easily that every point of $X$ is a supprim point. Another example of a RIM extension is an almost periodic homomorphism of minimal ttgs, which has a unique section and every point is a supprim point. For more details on RIM extensions see [9]. RIM extensions of minimal ttgs turn out to behave nicely with respect to the interpolation of maximal almost periodic factors, i.e. with respect to the equicontinuous structure relation. 
(1.1) TheOREM $([11,2.2])$. Let $\phi: \mathscr{X} \rightarrow \mathscr{Y}$ be a RIM extension, then $\phi$ is open in the supprim points.

In [11] a technique is developed to investigate the equicontinuous structure relation for RIM extensions. The most important results are 1.2 and its consequences 1.3 and 1.4 below.

(1.2) THEOREM. Let $\phi: \mathscr{X} \rightarrow \mathscr{Y}$ be a homomorphism of minimal ttgs, and let $\psi: \mathscr{X} \rightarrow \mathscr{Y}$ be a RIM extension with section $\lambda$ ( $\mathscr{Z}$ not necessarily minimal). Let $x \in X$ and let $V$ be an open set in $Z$. Then

$$
E_{\phi}[x] \times\left(V \cap \operatorname{supp} \lambda_{\phi(x)}\right) \subseteq \overline{T\left(\{x\} \times V \cap R_{\phi \psi}\right)} .
$$

(1.3) CoRollary. Let $\phi: \mathscr{X} \rightarrow \mathscr{Y}$ be a RIM extension of minimal ttgs with section $\lambda$. Then for every $x \in X$ with $x \in \operatorname{supp} \lambda_{\phi(x)}$ we have the equality $E_{\phi}[x]=Q_{\phi}[x]$. In particular, if a minimal ttg $\mathscr{X}$ has an invariant measure then $E_{\mathscr{P}}=Q_{\mathscr{X}}$.

(1.4) Corollary. Let $\phi: \mathscr{X} \rightarrow \mathscr{Y}$ be a RIM extension of minimal ttgs. Then

$$
E_{\phi}=Q_{\phi} \circ P_{\phi}=P_{\phi} \circ Q_{\phi}=\left\{\left(x_{1}, x_{2}\right) \in R_{\phi} \mid\left(u x_{1}, u x_{2}\right) \in Q_{\phi} \quad \text { for some } u \in J\right\} \text {. }
$$

Two other types of extensions that behave nicely with respect to the equicontinuous structure relation are the Bc extensions and the RIC extensions.

Let $\phi: \mathscr{X} \rightarrow \mathscr{Z}$ and $\psi: \mathscr{Y} \rightarrow \mathscr{Z}$ be surjective homomorphisms of ttgs (not necessarily minimal). Then the pair $(\phi, \psi)$ is said to satisfy the generalized Bronstein condition ( $\mathrm{gBc}$ ) if $\overline{J R_{\phi \psi}}=R_{\phi \psi}$; i.e. if the almost periodic points are dense in $R_{\phi \psi}$. If $\overline{J R_{\phi}}=R_{\phi}$ then $\phi$ is said to satisfy the Bronstein condition $(\mathrm{Bc})$; we shall also say that $\phi$ is a $\mathrm{Bc}$ map or a Bc extension. We say that $\phi$ satisfies the $n$-fold Bronstein condition for certain $n \in \mathbb{N}$ whenever

$$
R_{\phi}^{n}:=\left\{\left(x_{1}, \ldots, x_{n}\right) \in X^{n} \mid \phi\left(x_{1}\right)=\cdots=\phi\left(x_{n}\right)\right\}
$$

has a dense subset of almost periodic points (notation: $\phi$ is $n-\mathrm{Bc}$ ). So $\phi$ is a $\mathrm{Bc}$ map iff $\phi$ is $2-B c$.

A homomorphism $\phi: \mathscr{Z} \rightarrow \mathscr{Z}$ of minimal ttgs is called a RIC extension iff $\phi^{-}(z)=$ $u \circ u \phi^{*}(z)$ for every $z \in Z$ and for every $u \in J_{z}$ (RIC stands for Relatively InContractible). Note that a RIC extension is an open extension ([10, X.1.1]). Examples of RIC extensions are almost periodic, distal, and open point distal maps.

The proof of the next lemma is straightforward and will be omitted.

(1.5) Lemma. Let $\mathscr{X}$ be minimal. Consider the diagram of surjective homomorphisms:

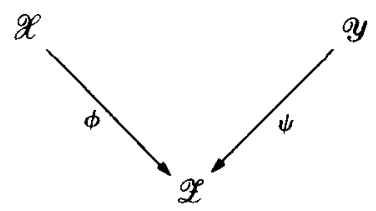

The following statements are equivalent:

(a) $\phi$ and $\psi$ satisfy $\mathrm{gBc}$;

(b) $R_{\phi \psi}=\overline{T\left(\{x\} \times u \psi^{\leftarrow} \phi(x)\right)}$ for some $x \in X$ and some $u \in J_{x}$;

(c) $R_{\phi \psi}=\overline{T\left(\{x\} \times u \psi^{\leftarrow} \phi(x)\right)}$ for every $x \in X$ and every $u \in J_{x}$. 
Two other useful remarks concerning dense sets of almost periodic points are:

(1.6) Remark. (Hypotheses as in 1.5.) Let $u \in J$. Then $\phi$ and $\psi$ satisfy $g B c$ iff

$$
\psi^{\leftarrow} \phi(x)=J_{x} \circ u \psi^{\leftarrow} \phi(x) \quad \text { for every } x \in X \text {. }
$$

In particular, $\phi$ is a $B c$ extension iff $\phi^{\leftarrow} \phi(x)=J_{x} \circ \phi^{-} \phi(x)$ for every $x \in X$.

Proof. The 'if' part is obvious.

Conversely, let $x \in X$ and $y \in \psi^{+} \phi(x)$. Then, by $1.5 \mathrm{c},(x, y)=\lim t_{i}\left(u x, u y_{i}\right)$ where $u y_{i} \in u \psi^{\leftarrow} \phi(u x)=u \psi^{\leftarrow} \phi(x)$. After passing to a suitable subnet let $p=\lim t_{i} u \in M$. Then it follows that

$$
y=\lim t_{i} u y_{i} \in p \circ u \psi^{\leftarrow} \phi(x) \text { and } p x=x .
$$

For $v \in J$ with $v p=p$ we have $v \in J_{x}$ and $y \in p \circ u \psi^{\leftarrow} \phi(x)=p \circ u p^{-1} u p \psi^{\leftarrow} \phi(x) \subseteq v \circ u p \psi^{\leftarrow} \phi(x) \subseteq v \circ u \psi^{\leftarrow} \phi(p x)=v \circ u \psi^{\leftarrow} \phi(x)$. So $y \in v \circ u \psi^{\leftarrow} \phi(x) \subseteq J_{x} \circ u \psi^{\star} \phi(x)$.

(1.7) REMARK. Let $\phi: \mathscr{X} \rightarrow \mathscr{Y}$ be a homomorphism of ttgs with $\mathscr{Y}$ minimal and $\mathscr{X}$ having a dense subset of almost periodic points. Then $\phi$ is semi-open (i.e., for every non-empty open $U \subseteq X, \phi[U]$ has a non-empty interior in $Y$ ).

Proof. The remark is well known for $\mathscr{X}$ minimal. Let $\mathscr{X}$ have a dense subset of almost periodic points and let $U \subseteq X$ be open and non-empty. Then $U \cap Z \neq \varnothing$ for some minimal orbit closure $Z$ in $X$. As $\phi[U \cap Z]$ has a non-empty interior in $Y$ the remark follows.

For the following denote by $\left(u \phi^{-}(z)\right)^{n}$ the cartesian $n$-power of $u \phi^{\leftarrow}(z)$; and by $2_{\phi}^{\mathscr{E}}$ the 'relativized hyper ttg', defined as a subttg of $2^{\mathscr{x}}$ by

$$
2_{\phi}^{X}:=\left\{A \in 2^{X} \mid \phi[A] \text { is a singleton }\right\} \text {. }
$$

The simple proof of the following lemma is left to the reader.

(1.8) LEMMA. Let $\phi: X \rightarrow \mathscr{Z}$ be a homomorphism of minimal ttgs.

(a) $\phi$ is $n$ - Bc iff $R_{\phi}^{n}=\operatorname{cl}_{X^{n}}\left[T .\left(u \phi^{\leftarrow}(z)\right)^{n}\right]$ for every $z \in Z$ and every $u \in J_{z}$.

(b) If $\phi$ is $n$ - Bc for all $n \in \mathbb{N}$, then for every $z \in Z$ and every $u \in J_{z}$ the set

is dense in $2_{\phi}^{X}$.

$$
\bigcup\left\{t\left\{x_{1}, \ldots, x_{n}\right\} \mid x_{i} \in u \phi^{*}(z), n \in \mathbb{N}, t \in T\right\} \subseteq 2_{\phi}^{X}
$$

(1.9) Theorem. Let $\phi: \mathscr{X} \rightarrow \mathscr{Z}$ be a homomorphism of minimal ttgs. The following statements are equivalent:

(a) $\phi$ is a RIC extension;

(b) $\phi \perp \psi$ for every proximal extension $\psi$ : $\mathscr{Y} \rightarrow \mathscr{Z}$ of minimal ttgs;

(c) $\phi$ and $\psi$ satisfy $\mathrm{gBc}$ for every $\psi: \mathscr{Y} \rightarrow \mathscr{Z}$ with $\mathscr{Y}$ having a dense subset of almost periodic points;

(d) $\phi$ is open and $n-B c$ for every $n \in \mathbb{N}$.

Proof. (a) and (b) are equivalent by [10, X.1.3].

(a) $\Rightarrow$ (c) Let $U \times V \cap R_{\phi \psi}$ be a non-empty (basic) open set in $R_{\phi \psi}$. As $\phi$ is open and $\psi$ is semi-open (1.7),

$$
W:=\psi\left[\psi^{\leftarrow} \phi[U] \cap V\right]^{\circ}
$$


is a non-empty open subset of $Z$ and $\phi\left[U^{\prime}\right]=\psi\left[V^{\prime}\right]$, where $U^{\prime}:=U \cap \phi^{+}[W]$ and $V^{\prime}:=V \cap \psi^{-}[W]$.

Let $y \in V^{\prime}$ be an almost periodic point, say $y=v y$ for certain $v \in J$, and let $x \in U^{\prime} \cap \phi^{-} \psi(v y)$. As $\phi$ is RIC, $x \in v \circ v \phi^{\leftarrow} \psi(v y)$ so $x=\lim t_{i} v x_{i}$ for some net $t_{i} \rightarrow v$ and $v x_{i} \in \phi^{\leftarrow} \psi(v y)$. Hence $(x, y)=\lim t_{i}\left(v x_{i}, v y\right)$, and for certain $i_{0}$

$$
t_{i_{0}}\left(v x_{i_{0}}, v y\right) \in U^{\prime} \times V^{\prime} \cap R_{\phi \psi} \subseteq U \times V \cap R_{\phi \psi} .
$$

Consequently $R_{\phi \psi}$ has a dense subset of almost periodic points.

(c) $\Rightarrow$ (d) Follows by induction from the observation that $R_{\phi}^{n+1} \cong R_{\phi \eta}$, where $\eta: \mathscr{R}_{\phi}^{n} \rightarrow \mathscr{Z}$ is the restriction of $\phi^{n}: \mathscr{Z}^{n} \rightarrow \mathscr{Z}^{n}$.

(d) $\Rightarrow$ (a) Let $z \in Z$ and $u \in J_{z}$. By $1.8 \mathrm{~b}$, there are sets $t_{i}\left\{x_{1}^{i}, \ldots, x_{n_{i}}^{i}\right\}$ with $x_{j}^{i} \in$ $u \phi^{\leftarrow}(z)$ which in $2_{\phi}^{X}$ converge to $\phi^{-}(z)$. As

$$
t_{i}\left\{x_{1}^{i}, \ldots, x_{n_{i}}^{i}\right\}=t_{i} u\left\{x_{1}^{i}, \ldots, x_{n_{i}}^{i}\right\} \subseteq t_{i} u \circ u \phi^{\leftarrow}(z),
$$

it follows that

$$
\phi^{\leftarrow}(z)=\lim t_{i}\left\{x_{1}^{i}, \ldots, x_{n_{i}}^{i}\right\} \subseteq \lim t_{i} u \circ u \phi^{\leftarrow}(z)=p \circ u \phi^{\leftarrow}(z)
$$

where $p=\lim t_{i} u \in M$ (after passing to a suitable subnet). But, clearly, $p^{\circ} u \phi^{\leftarrow}(z) \subseteq$ $\phi^{-}(p z)$, so $z=p z$ and $z=u p^{-1} z$. By openness of $\phi$ we know that $\phi^{-}(z)=$ $u p^{-1} \circ \phi^{\leftarrow}(z)$, so

$$
\phi^{-}(z)=u p^{-1} \circ \phi^{-}(z) \subseteq u p^{-1} \circ p \circ u \phi^{-}(z)=u \circ u \phi^{-}(z)
$$

Obviously, $u \circ u \phi^{\leftarrow}(z) \subseteq \phi^{+}(z)$, which shows that $\phi^{\leftarrow}(z)=u \circ u \phi^{\leftarrow}(z)$.

There are several ways to study the equicontinuous structure relation for $\mathrm{Bc}$ extensions:

(i) Elementary, using some trickery with syndetic sets and the uniform structure. In [2, th 3], it was proved that $E_{\phi}=Q_{\phi}$ for open Bc extensions $\phi$ of minimal ttgs, so certainly for RIC extensions. A suitable 'shadow diagram' ([7]) finishes the general $\mathrm{Bc}$ case.

(ii) Using our knowledge about (open) RIM extensions ([11], [16]) and a(nother) shadow diagram ([9]).

(iii) The method of the $\mathfrak{F}$-topologies ([8], [4], [7] and [15]).

With help of the $\mathfrak{F}$-topologies one tries to imitate the properties of a compact group action. We shall briefly describe the $\mathfrak{F}$-topologies and some of their properties, resulting in a first description of the equicontinuous structure relation $(1.16,1.17)$. Our approach will be based upon [15] which will also serve as a general reference.

First we shall specify a certain neighbourhood base for $u$ in $M$ (for the usual topology). Let $V$ be a subset of $T$ such that $u \in$ int $_{S_{T}} \operatorname{cl}_{S_{T}}[V]$, and let $h(V):=$ $\operatorname{cl}_{S_{T}}[V] \cap M$. Then define

$$
V(u):=\left\{t \in T \mid t u \in \text { int }_{M} h(V)\right\} .
$$

Clearly, $V(u)$ is open in $T$, but in general $V$ and $V(u)$ do not coincide. However, the collection of subsets $V$ of $T$ for which $V$ and $V(u)$ are the same can be used to define a neighbourhood base for $u$ in $M$, as follows. 
(1.10) LEMMA. The collection $\left\{h(V) \mid V=V(u) \subseteq T, u \in\right.$ int $\left._{s_{T}} \mathrm{cl}_{S_{T}}[V]\right\}$ forms a neighbourhood base for $u$ in $M$.

Let $\mathscr{X}$ be a ttg. We shall define a topology $\mathfrak{\xi}(\mathscr{X}, u)$ on $u X=\{x \in X \mid u x=x\}$ by specifying a neighbourhood base for every $x=u x$ in $u X$. So let $x \in u X$. A typical neighbourhood of $x$ in $(u X, \mathfrak{F}(\mathscr{X}, u))$ will be a set of the form

$$
[U, V] \cap u X \quad \text { with }[U, V]:=\bigcup\left\{t^{-1} U \mid t \in V\right\},
$$

where $U$ is a neighbourhood of $x$ in $X$ (usual topology) and $V$ is an open subset of $T$ such that $u \in$ int $_{S_{T}} \mathrm{cl}_{S_{T}}[V]$ and $V=V(u)$. The union of those neighbourhood bases forms a base for the $\mathfrak{F}(\mathscr{X}, u)$-topology on $u X$.

(1.11) Properties. (a) The $\mathfrak{F}(\mathscr{X}, u)$-topology can be defined by the closure operator $A \mapsto u(u \circ A),(A \subseteq u X)$.

(b) $(u X, \mathfrak{F}(\mathscr{X}, u))$ is a compact $T_{1}$ topological space;

(c) $\lambda_{a}: x \mapsto a x:(u X, \mathfrak{F}(\mathscr{X}, u)) \rightarrow(u X, \mathfrak{F}(\mathscr{X}, u))$ is a homeomorphism for every $a \in u M$

(d) $\lambda_{v}: x \mapsto v x:(u X, \mathfrak{F}(\mathscr{X}, u)) \rightarrow(v X, \mathfrak{F}(\mathscr{X}, v))$ is a homeomorphism for every $v \in J$. A special case is $(u M, \mathfrak{F}(\mathfrak{M}, u))$. With the $\mathfrak{F}(\mathfrak{M}, u)$-topology $u M$ is a group with a compact $T_{1}$ underlying space and not only the left translations are homeomorphisms (1.11b) but also the right translations and the inversion are. This space $(u M, \mathfrak{F}(\mathfrak{M}, u)$ ) is some sort of prototype for the $\mathfrak{F}$-topologies; i.e. we can consider the $\mathfrak{F}$-topologies as quotients of the $\mathfrak{F}(\mathfrak{M}, u)$-topology.

(1.12) THEOREM. Let $\phi: \mathscr{X} \rightarrow \mathscr{Y}$ be a homomorphism of minimal ttgs and define the map $\phi_{u}$ by

Then

$$
\phi_{u}:=\left.\phi\right|_{u X}:(u X, \mathfrak{F}(\mathscr{X}, u)) \rightarrow(u Y, \mathfrak{F}(\mathscr{Y}, u))
$$

(a) $\phi_{u}$ is a homeomorphism iff $\phi$ is proximal;

(b) $\phi_{u}$ is a closed continuous surjection;

(c) $\phi_{u}$ is open.

Proof. (a) [15, 2.5.8].

(b) $[15,2.5 .7]$.

(c) It is well known (e.g. $[10, X .3 .2]$ ) that there are proximal maps $\sigma$ and $\tau$ and a RIC extension $\phi^{\prime}$ such that $\tau^{\circ} \phi^{\prime}=\phi^{\circ} \sigma$, ([EGS diagram]). From (a) it follows that it suffices to show that $\phi_{u}^{\prime}$ is $\mathfrak{F}$-open; or better, it follows that we only have to prove the statement for RIC extensions.

Let $\phi$ be a RIC extension, $x \in u X$ and let $[U, V] \cap u X$ be an $\mathfrak{F}(\mathscr{X}, u)$-neighbourhood of $X$ in $u X$ with $V=V(u)$ open in $T$ and $U$ is a neighbourhood of $X$ in $X$. As $\phi$ is open, it will follow that $\phi_{u}$ is open. First note that

$$
\phi_{u}[[U, V] \cap u X] \subseteq \phi[[U, V]] \cap \phi[u X]=[\phi[U], V] \cap u Y .
$$

Let $y=u y \in[\phi[U], V] \cap u Y$, then $y=\phi\left(t^{-1} x^{\prime}\right)$ for some $t \in V$ and $x^{\prime} \in U$. As $\phi$ is RIC we have $z:=t^{-1} x^{\prime} \in \phi^{\leftarrow}(y)=u \circ u \phi^{\leftarrow}(y)$. Let $\left\{t_{i}\right\}_{i}$ be a net in $T$ with $t_{i} \rightarrow u$ and let $x_{i} \in u \phi^{\leftarrow}(y)$ be such that $z=\lim t_{i} x_{i}$. Since left multiplication with $t$ is a homeomorphism we have $t t_{i} x_{i} \rightarrow t z=x^{\prime}$ and $t t_{i} \rightarrow t u$, hence $t t_{i} u \rightarrow t u$. As $t \in V=V(u)$ we 
have $t u \in \operatorname{int}_{M}\left(\mathrm{cl}_{S_{T}}[V] \cap M\right)$, so $t t_{i} u \in \operatorname{int}_{M}\left(\mathrm{cl}_{S_{T}}[V] \cap M\right)$ eventually, hence $t t_{i} \in$ $V(u)=V$ eventually. Also $t t_{i} x_{i} \in U$ eventually, so we can find some $i_{0}$ such that $t t_{i_{0}} x_{i_{0}} \in U$ and $t t_{i_{0}} \in V$. This shows that

$$
x_{i_{0}}=\left(t t_{i_{0}}\right)^{-1} \cdot t t_{i_{0}} x_{i_{0}} \in V^{-1} U
$$

so $x_{i_{0}} \in[U, V] \cap u \phi^{\leftarrow}(y)$. Hence $x_{i_{0}} \in[U, V] \cap u X$, while $\phi\left(x_{i_{0}}\right)=y$ and so it follows that $y \in \phi_{u}[[U, V] \cap u X]$, which implies

$$
\phi_{u}[[U, V] \cap u X]=[\phi[U], V] \cap u Y,
$$

in the case where $\phi$ is a RIC extension. (Compare [7, Cor. 3.3.] for another approach.)

As every minimal ttg $\mathscr{X}$ is a factor of $\mathfrak{M}$, it follows from 1.12 that $(u X, \mathfrak{F}(\mathscr{X}, u))$ is an open, closed and continuous image of $(u M, \mathfrak{F}(\mathfrak{M}, u))$. So $(u M, \mathfrak{F}(\mathfrak{M}, u))$ plays a central role in the observations about $\mathfrak{\wp}$-topologies.

Let $\mathscr{X}$ be a minimal ttg, $x_{0}=u x_{0} \in X$. Then define the Ellis group $\mathscr{G}\left(\mathscr{X}, \varkappa_{0}\right)$ of $\mathscr{X}$ with respect to $x_{0}$ by

$$
\mathbb{G}\left(\mathscr{X}, x_{0}\right)=\left\{a \in u M \mid a x_{0}=x_{0}\right\} \quad\left(=u M \cap \rho_{x_{0}}^{\leftarrow}\left(x_{0}\right)\right) .
$$

Clearly, $\mathscr{B}\left(\mathscr{X}, x_{0}\right)$ is a subgroup of $u M$. As $\rho_{x_{0}}: a \mapsto a x_{0}: \mathfrak{M} \rightarrow \mathscr{X}$ is a homomorphism of minimal ttgs and $(u X, \mathfrak{F}(\mathscr{X}, u))$ is $\mathrm{T}_{1}$, it follows from $1.12 \mathrm{~b}$ that $\mathscr{G}\left(\mathscr{X}, x_{0}\right)$ is $\mathfrak{S}(\mathfrak{M}, u)$-closed. Note that, by $: .12 \mathrm{a}, \phi: \mathscr{X} \rightarrow \mathscr{Y}$ is proximal iff $\left(\mathscr{S}\left(\mathscr{X}, u x_{0}\right)=\right.$ (S) $\left(\mathscr{Y}, \phi\left(u x_{0}\right)\right)$.

On the other hand, if $F$ is an $\mathfrak{\Im}(\mathfrak{M}, u)$-closed subgroup of $u M$, then there exists a minimal ttg $\mathfrak{U}(\Gamma)$ defined by

$$
\mathfrak{A}(F):=\{p \circ F \mid p \in M\} \subseteq 2^{M},
$$

such that $F=\mathfrak{S}(\mathfrak{U}(F), u \circ F)$, i.e. $F$ is the Ellis group of $\mathfrak{U}(F)$ with respect to $u \circ F$. (1.13) The $\operatorname{ttg} \mathfrak{A}(F)$ is the universal minimal proximal extension of every minimal $\operatorname{tg} \mathscr{X}$ with Ellis group $F$. So let $x_{0}=u x_{0} \in X$ be such that $\mathscr{B}\left(\mathscr{X}, x_{0}\right)=F$, then $\sigma: p \circ F \mapsto p x_{0}: \mathfrak{U}(F) \rightarrow \mathscr{X}$ is the maximally proximal extension of $\mathscr{X}$. Note that every extension $\psi: \mathscr{Y} \rightarrow \mathfrak{U}(F)$ is a RIC extension (use $1.9 \mathrm{~b}$ and the universality of $\mathscr{U}(F)$ ).

Let $\phi: \mathscr{X} \rightarrow \mathscr{Y}$ be a homomorphism of minimal ttgs, $x \in u X, y=\phi(x)$ and $F=(\mathscr{S}(\mathscr{Y}, y)$. Then $u \phi^{\leftarrow}(y)=F x$. Let $\mathfrak{N}_{x}$ denote the $\mathfrak{F}(\mathscr{X}, u)$-neighbourhood system of $x$ in $F x$. Define ([15])

$$
E(x):=E(x, \phi, u)=\bigcap\left\{\mathrm{cl}_{\tilde{F}(\mathscr{X}, u)} U \mid U \in \mathfrak{N}_{x}\right\} .
$$

For $\rho_{y}: p \mapsto p y: \mathfrak{M} \rightarrow \mathscr{Y}$ we have $\mathrm{H}(F):=E\left(u, \rho_{y}, u\right)$ is the smallest $\mathfrak{F}(\mathfrak{M}, u)$-closed normal subgroup $K$ of $F$ such that $F / K$ is a $C_{2}$ topological group.

By 1.12 , it follows easily that $E(x)=\mathrm{H}(F) x$. It turns out that $\left\{E\left(x^{\prime}\right) \mid x^{\prime} \in F x\right\}$ forms a partition of $u \phi^{\leftarrow}(x)$ and that $F / \mathrm{H}(F)$ acts on it as a $\mathrm{CT}_{2}$ topological group. This is what we meant by imitating the compact group action (discussion after 1.9). Paraphrazed, we may say that in $H(F) x$ we collect all the non-equicontinuous garbage for $\phi$ in $u \phi^{\leftarrow}(y)$, which might be illustrated by the following theorem $([6$, $5.4,6.3])$. 
(1.14) TheOREM. Let $\phi: \mathscr{X} \rightarrow \mathscr{Y}$ be a Bc extension of minimal ttgs, $x \in u X$ and $F=\mathfrak{G}(\mathscr{Y}, \phi(x))$. Then $E_{\phi}[x]=J_{\phi(x)} \mathrm{H}(F) x,\left(\right.$ so $\left.\mathscr{G}\left(\mathscr{X} / E_{\phi}, E_{\phi}[x]\right)=\mathrm{H}(F) \mathscr{G}(\mathscr{X}, x)\right)$.

The next lemma is a slightly modified version of a crucial idea in [15].

(1.15) Lemma. Let $\phi: \mathscr{X} \rightarrow \mathscr{Y}$ be a homomorphism of minimal ttgs, $x \in u X, F=$ $\mathfrak{S}(\mathscr{Y}, \phi(x))$, and denote by $\mathfrak{R}_{x}$ the collection of $\mathfrak{F}(\mathscr{X}, u)$-neighbourhoods of $x$ in $u \phi^{\leftarrow} \phi(x)=F x$. Then

$$
u \circ F x \cap J \mathrm{H}(F) x \subseteq u \circ U \quad \text { for every } U \equiv \mathfrak{R}_{x} .
$$

Proof. Let $U$ be an open $\mathfrak{F}(\mathscr{X}, u)$-neighbourhood of $x$ in $F x$. Then $O=\{f \in F \mid f x \in U\}$ is an $\mathfrak{F}(\mathfrak{M}, u)$-open neighbourhood of $u$ in $F$, so $O \cap O^{-1}$ is an $\mathfrak{F}(\mathfrak{M}, u)$-neighbourhood of $u$ in $F$, hence $V=\left(O \cap O^{-1}\right) x$ is an open $\mathfrak{F}(\mathscr{X}, u)$-neighbourhood of $x$ in $F x$ and $V \subseteq U$. Note that $V$ is symmetric in the sense that for $f \in F$ we have $f x \in V$ iff $f^{-1} x \in V$, and remark that $\operatorname{cl}_{\widetilde{F}(\mathscr{x}, u)} V$ is symmetric too.

Define $A:=\operatorname{int}_{\mathfrak{F}(\mathscr{X}, u)} \mathrm{cl}_{\mathfrak{F}(\mathscr{X}, u)} V$ in the relative $\mathfrak{\Im}(\mathscr{X}, u)$-topology on $F x$. We claim that

$$
\{A\} \cup\left\{g V \mid g \in F \text { and } g x \notin \mathrm{cl}_{\Re(\mathscr{x}, u)} V\right\}
$$

is an $\mathfrak{\mho}(\mathscr{X}, u)$-opening covering of $F x$, and prove it as follows:

Let $f \in F$ be such that $f x \notin A$; i.e.

$$
f x \in F x \backslash A=\operatorname{cl}_{\Re(x, u)}\left(F x \backslash \operatorname{cl}_{\tilde{r}(\mathscr{x}, u)} V\right) .
$$

So we can find a net $\left\{f_{i} x\right\}_{i}$ with $f_{i} x \in F x \backslash \mathrm{cl}_{\mathscr{F}(x, u)} V$ such that $f_{i} x \rightarrow f x$ in the $\mathfrak{F}(\mathscr{X}, u)$ topology. Since

$$
\lambda_{f^{-1}}:(F x ; \mathfrak{F}(\mathscr{X}, u)) \rightarrow(F x, \mathfrak{F}(\mathscr{X}, u))
$$

is a homeomorphism, $f^{-1} f_{i} x \rightarrow x$ in the $\mathfrak{F}(\mathscr{X}, u)$-topology. As $V \in \mathfrak{N}_{x}$, there is an $i_{0}$ with $f^{-1} f_{i_{0}} x \in V$ and by symmetry of $V, f_{i_{0}}^{-1} f x \in V$. Hence $f x \in f_{i_{0}} V$, where $f_{i_{0}} \in F$ is such that $f_{i_{0}} x \in F x \backslash \operatorname{cl}_{\widetilde{F}(\mathscr{X}, u)} V$, which establishes our claim.

By compactness, there are finitely many $g_{i} \in F$ with $g_{i} x \notin \operatorname{cl}_{\mathfrak{F}(\mathscr{x}, u)} V$, say $g_{1}, \ldots, g_{n}$, such that

$$
F x \subseteq A \cup \bigcup\left\{g_{i} V \mid i \in\{1, \ldots, n\}\right\}
$$

As $\{A\} \cup\left\{g_{i} V \mid i \in\{1, \ldots, n\}\right\}$ is a finite collection it follows that

$$
\boldsymbol{u} \circ F x=u \circ\left(A \cup \bigcup\left\{g_{i} V \mid i \in\{1, \ldots, n\}\right\}\right)=u \circ A \cup \bigcup\left\{u \circ g_{i} V \mid i \in\{1, \ldots, n\}\right\} .
$$

Now let $x^{\prime} \in J H(F) x \cap u \circ F x$, say $x^{\prime}=v p x$ for some $v \in J$ and $p \in H(F)$. We shall prove that $x^{\prime}=v p x \notin u \circ g_{i} V$ for every $i \in\{1, \ldots, n\}$. It then follows that

$$
x^{\prime} \in u \circ A \subseteq u \circ \operatorname{cl}_{\mathfrak{F}(\mathscr{x}, u)} V=u \circ u(u \circ V) \subseteq u \circ V \text {, }
$$

which proves the theorem. Suppose $v p x \in u \circ g_{i} V$, then

$$
\begin{aligned}
x= & u x=u p^{-1} v p x \in u p^{-1}\left(u \circ g_{i} V\right) \subseteq u p^{-1}\left(u \circ u p u p^{-1} g_{i} V\right) \\
& \subseteq u\left(u p^{-1} \circ u p \circ u p^{-1} g_{i} V=u\left(u \circ u p^{-1} g_{i} V\right)=\operatorname{cl}_{\mathfrak{F}(\mathscr{R}, u)} u p^{-1} g_{i} V .\right.
\end{aligned}
$$

As $\mathrm{H}(F)$ is a normal subgroup of $F$ and $g_{i} \in F$ we can find $q \in \mathrm{H}(F)$ such that $u p^{-1} g_{i}=g_{i} q$, so

$$
x \in \mathrm{cl}_{\mathfrak{F}(\mathscr{X}, u)} g_{i} q V=g_{i} \mathrm{cl}_{\mathfrak{F}(\mathscr{X}, u)} q V \subseteq g_{i} \mathrm{cl}_{\mathfrak{F}(\mathscr{X}, u)} \mathrm{H}(F) V .
$$




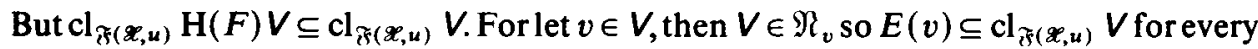
$v \in V$; as $E(v)=\mathrm{H}(F) v, \mathrm{H}(F) V \subseteq \operatorname{cl}_{\mathfrak{F}(\mathscr{R}, u)} V$ and consequently $\mathrm{cl}_{\mathfrak{R}(\mathscr{X}, u)} \mathrm{H}(F) V \subseteq$ $\mathrm{cl}_{\mathfrak{F}(\mathscr{x}, u)} V$. This shows that $x \in g_{i} \operatorname{cl}_{\mathfrak{F}(\mathscr{x}, u)} V$ and so $g_{i}^{-1} x \in \mathrm{cl}_{\mathfrak{F}(\mathscr{x}, u)} V$. By symmetry of $\operatorname{cl}_{\mathfrak{F}(\mathscr{X}, u)} V, g_{i} x \in \mathrm{cl}_{\mathfrak{F}(\mathscr{x}, u)} V$, which contradicts the choice of $g_{i}$.

Now we are ready for the main theorem of this section.

(1.16) Theorem. Let $\phi: \mathscr{X} \rightarrow \mathscr{Y}$ be a homomorphism of minimal ttgs, $x \in u X$ and $F=\mathbb{B}(\mathscr{Y}, \phi(x))$.

(a) If $\phi$ is a RIC extension then $E_{\phi}[x] \subseteq u \circ U$ for every $U \in \mathfrak{N}_{x}([15,2.6 .1])$.

(b) If $\phi$ is a $\mathrm{Bc}$ extension then $E_{\phi}[x] \subseteq J_{x^{\prime}} \cup U$ for every $x^{\prime} \in \phi^{\leftarrow} \phi(x)$ and every $U \in \mathfrak{R}_{\boldsymbol{x}}$.

Proof. As the proof of (a) is similar to that of (b), we just prove (b).

(b) Let $U \in \mathfrak{N}_{x}$ and let $x^{\prime} \in \phi^{\leftarrow} \phi(x)=\phi^{\leftarrow} \phi\left(x^{\prime}\right)$. By 1.6, $\phi^{\leftarrow} \phi(x)=J_{x^{\circ}} u \phi^{\leftarrow} \phi(x)$; so from 1.14 it follows that

$$
E_{\phi}[x] \subseteq J_{x^{\circ}} \circ F x \cap J_{\phi(x)} \mathrm{H}(F) x .
$$

Let $z \in E_{\phi}[x]$, say $z \in v \circ F x \cap J H(F) x$ for certain $v \in J_{x^{\prime}}$. Note that

$$
v \circ F x=v \circ u v F x \subseteq v u \circ v F x=v \circ v F x=v \circ v u F x \subseteq v \circ u F x=v \circ F x \text {, }
$$

so $v \circ F x=v \circ v F x=v \circ v F v x$ and $z \in v \circ v F v x \cap J v \mathrm{H}(F) v x$. Applying 1.15 to $v x$ it follows that

$$
v \circ v F x \cap J v \mathrm{H}(F) v x \subseteq v \circ v U \text {. }
$$

As $v \circ v U=v \circ u U=v \circ U$ (similar to $v \circ F x=v \circ v F x$ ), we may conclude that

$$
z \in v \circ F x \cap J \mathrm{H}(F) x=v \circ v F x \cap J v \mathrm{H}(F) v x \subseteq v \circ v U=v \circ U .
$$

Consequently $E_{\phi}[x] \subseteq J_{x^{\circ}} \circ U$.

(1.17) Corollary. Let $\phi: \mathscr{X} \rightarrow \mathscr{Y}$ be a Bc extension. Then $E_{\phi}=Q_{\phi}$.

Proof. Let $x \in X$, and $u \in J_{x}$. By $1.16 \mathrm{~b}$ with $x^{\prime}=x$ we know that $E_{\phi}[x] \subseteq J_{x} \circ U$ for every $U \in \mathfrak{N}_{x}$. Let $\alpha \in \mathcal{U}_{X}$ and let $U$ be a neighbourhood of $x$ in $X$ (usual topology) such that $U \times U \subseteq \alpha$. By 1.10 , there is a $V=V(u)$ open in $T$ such that $V x \subseteq U$. Define $V:=[U, V] \cap u \phi^{\leftarrow} \phi(x)$. Then

$$
\{x\} \times E_{\phi}[x] \subseteq\{x\} \times J_{x} \circ V=J_{x} \circ(\{x\} \times V) .
$$

As, clearly, $\{x\} \times V \subseteq V^{-1} \cdot\left(V x \times U \cap R_{\phi}\right) \subseteq T \alpha \cap R_{\phi}$, it follows that

$$
\{x\} \times E_{\phi}[x] \subseteq J_{x} \circ(\{x\} \times V) \subseteq \overline{T \alpha \cap R_{\phi}} \text {. }
$$

Since $\alpha \in \mathcal{U}_{X}$ was arbitrary, we have $\{x\} \times E_{\phi}[x] \subseteq Q_{\phi}$, so $E_{\phi}[x] \subseteq Q_{\phi}[x]$. But $x$ and $u \in J_{x}$ were arbitrary in the discussion up to now, so it follows that $E_{\phi}=Q_{\phi}$.

We can do better than the corollary above as will be shown in 3.7.

\section{Revitalized weak disjointness}

In this section we relate weak disjointness of maps and that of their maximally almost periodic factors. For that we need to keep control over open sets, which can be done by means of a fair amount of openness in the maps involved. 
(2.1) LEMMA. Consider the following commutative diagram of surjective homomorphisms of ttgs:

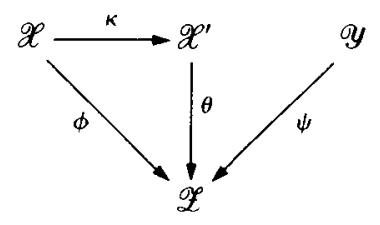

and let $\eta: R_{\phi \psi} \rightarrow Z$ be the obvious map $(\eta(x, y)=\phi(x)=\psi(y))$.

(a) If one of $\phi, \psi$ is open and the other is semi-open then $\eta$ is semi-open, and for every non-empty open $W \subseteq R_{\phi \psi}$ there are open sets $U$ and $V$ in $X$ and $Y$ with

$$
\varnothing \neq U \times V \cap R_{\phi \psi} \subseteq W \text { and } \phi[U]=\psi[V] .
$$

(b) If $\eta$ and $\kappa$ are semi-open then $\kappa \times \mathrm{id}_{Y}: R_{\phi \psi} \rightarrow R_{\theta \psi}$ is semi-open, and for every open $W \subseteq R_{\phi \psi}$ there are $U$ and $V$ as in (a).

Proof. (a) Without loss of generality let $\psi$ be open and $\phi$ semi-open and let $W \subseteq R_{\phi \psi}$ be a non-empty open set in $R_{\phi \psi}$. Let $U^{\prime}$ and $V^{\prime}$ be open in $X$ and $Y$ such that

$$
\varnothing \neq U^{\prime} \times V^{\prime} \cap R_{\phi \psi} \subseteq W .
$$

Then $U^{\prime} \cap \phi^{\leftarrow} \psi\left[V^{\prime}\right]$ is non-empty and open so by semi-openness of $\phi, O:=$ $\phi\left[U^{\prime} \cap \phi^{\leftarrow} \psi\left[V^{\prime}\right]\right]^{\circ}$ is non-empty and open. Define $U:=U^{\prime} \phi^{\leftarrow}[O]$ and $V:=V^{\prime} \cap$ $\psi^{*}[O]$. Then as is easily seen,

(i) $O \subseteq \phi\left[U^{\prime}\right] \cap \psi\left[V^{\prime}\right] \subseteq \eta[W]$, so $\eta$ is semi-open;

(ii) $\varnothing \neq U \times V \cap R_{\phi \psi} \subseteq W$ and $\phi[U]=\psi[V]=O$;

which proves (a).

(b) Let $W$ be non-empty and open in $R_{\phi \psi}$ and let $U$ and $V$ be as in (a), with $O=\eta\left(U^{\prime} \times V^{\prime} \cap R_{\phi \psi}\right)^{\circ}$. Semi-openness of $\kappa$ implies $\kappa[U]^{\circ} \neq \varnothing$, and as $\phi[U]=\psi[V]$ it follows that

$$
\kappa[U]^{\circ} \times V \cap R_{\theta \psi} \neq \varnothing
$$

hence $\kappa \times \mathrm{id}_{Y}$ is semi-open.

In the following remark we collect some situations in which $\eta$ is semi-open and which are useful for our puposes. So consider the following diagram:

(2.2) Diagram

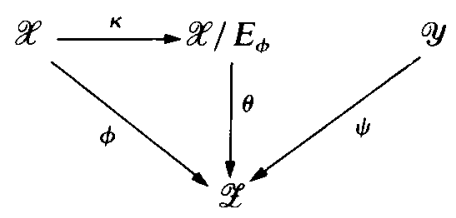

with $\mathscr{X}$ and $\mathscr{Z}$ minimal and $\mathscr{Y}$ not necessarily minimal.

(2.3) RemARK. Consider diagram 2.2. In each of the following cases $\eta: R_{\phi \psi} \rightarrow Z$ is semi-open (and, by minimality of $\mathscr{X}$, also $\kappa \times \mathrm{id}_{Y}: R_{\phi \psi} \rightarrow R_{\theta \psi}$ is semi-open).

(a) $\phi$ and $\psi$ satisfy $\mathrm{gBc}$;

(b) $\psi$ is open; 
(c) $\phi$ is open and $Y$ has a dense subset of almost periodic points;

(d) $\phi$ is open, $\psi$ is a RIM extension and $Y$ has a dense subset of supprim points.

Proof. (a), (b) and (c) are obvious from 1.7 (and 2.1).

(d) As $\psi$ is open in the supprim points, there is a dense subset of $Y$ in which $\psi$ is open, hence $\psi$ is semi-open.

(2.4) THEOREM. Consider diagram 2.2 and let $\phi$ and $\psi$ satisfy one of the conditions in 2.3. If for every non-empty (basic) open set $U \times V \cap R_{\phi \psi}$ there is an open set $\tilde{U}=E_{\phi}[\tilde{U}]$ in $X$ such that

then $\phi-\psi$ iff $\theta-\psi$.

$$
\varnothing \neq \tilde{U} \times V \cap R_{\phi \psi} \subseteq \overline{T\left(U \times V \cap R_{\phi \psi}\right)}
$$

Proof. As $\kappa \times \mathrm{id}_{Y}\left[R_{\phi \psi}\right]=R_{\theta \psi}, \phi-\psi$ implies $\theta-\psi$.

Conversely, suppose $\theta-\psi$. Let $U \times V \cap R_{\phi \psi}$ be a non-empty (basic) open set in $R_{\phi \psi}$ and let $\tilde{U}$ be as in the assumption. Clearly, as $\kappa \kappa[\tilde{U}]=\tilde{U}, \kappa[\tilde{U}]$ is open in $X / E_{\phi}$ and $\kappa[\tilde{U}] \times V \cap R_{\theta \psi} \neq \varnothing$. So, by ergodicity of $R_{\theta \psi}, T\left(\kappa[\tilde{U}] \times V \cap R_{\theta \psi}\right)$ is dense in $R_{\theta \psi}$. As $\kappa \times \mathrm{id}_{Y}$ is semi-open,

$$
\left(\kappa \times \mathrm{id}_{Y}\right)^{\leftarrow}\left[T\left(\kappa[\tilde{U}] \times V \cap R_{\theta \psi}\right)\right]
$$

is dense in $R_{\phi \psi}$. Hence, as

$$
\left(\kappa \times \operatorname{id}_{Y}\right)^{\leftarrow}\left[T\left(\kappa[\tilde{U}] \times V \cap R_{\theta \psi}\right)\right]=T\left(\tilde{U} \times V \cap R_{\phi \psi}\right) \subseteq \overline{T\left(U \times V \cap R_{\phi \psi}\right)},
$$

it follows that $R_{\phi \psi}=\overline{T\left(U \times V \cap R_{\phi \psi}\right)}$. Consequently, $R_{\phi \psi}$ is ergodic.

Now we shall look for situations in which the assumptions of 2.4 are satisfied. For that we need the following lemmas.

(2.5) Lemma. Consider diagram 2.2 and suppose that one of the conditions in 2.3 is satisfied. If every non-empty (basic) open set $U^{\prime} \times V^{\prime} \cap R_{\phi \psi}$ contains a point $(x, y)$ such that

$$
E_{\phi}[x] \times\{y\} \subseteq \overline{T\left(U^{\prime} \times V^{\prime} \cap R_{\phi \psi}\right)}
$$

then the assumption in 2.4 is satisfied.

Proof. We shall show that for a non-empty (basic) open set $U \times V \cap R_{\phi \psi}$ with $\phi[U]=\psi[V]$ the set $\tilde{U}=E_{\phi}[\tilde{U}]:=\kappa^{\leftarrow}\left[\kappa[U]^{\circ}\right]$ is such that

$$
\tilde{U} \times V \cap R_{\phi \psi} \subseteq \overline{T\left(U \times V \cap R_{\phi \psi}\right)} .
$$

As one of the conditions in 2.3 is satisfied, the lemma follows.

Let $U$ and $V$ be open in $X$ and $Y$ such that $\phi[U]=\psi[V]$, define $\tilde{U}:=\kappa^{\leftarrow}\left[\kappa[U]^{\circ}\right]$ and remark that $\tilde{U} \times V \cap R_{\phi \psi} \neq \varnothing$. Note that it is sufficient to show that for an arbitrary non-empty (basic) open subset

$$
U^{\prime} \times V^{\prime} \cap R_{\phi \psi} \subseteq \tilde{U} \times V \cap R_{\phi \psi}
$$

we have $U^{\prime} \times V^{\prime} \cap T\left(U \times V \cap R_{\phi \psi}\right) \neq \varnothing$. By assumption, there is a point $(x, y) \in$ $U^{\prime} \times V^{\prime} \cap R_{\phi \psi}$ such that

$$
E_{\phi}[x] \times\{y\} \subseteq \overline{T\left(U^{\prime} \times V^{\prime} \cap R_{\phi \psi}\right)}
$$


As $U^{\prime} \subseteq \tilde{U}=\kappa^{-}\left[\kappa[U]^{\circ}\right]$ there is an $x^{\prime} \in U$ such that $x \in \kappa^{\leftarrow} \kappa\left(x^{\prime}\right)=E_{\phi}\left[x^{\prime}\right]$, so $x^{\prime} \in$ $E_{\phi}[x]$. But then

$$
\left(x^{\prime}, y\right) \in U \times V \cap E_{\phi}[x] \times\{y\} \subseteq U \times V \cap \overline{T\left(U^{\prime} \times V^{\prime} \cap \overline{R_{\phi \psi}}\right)},
$$

so $U \times V \cap T\left(U^{\prime} \times V^{\prime} \cap R_{\phi \psi}\right) \neq \varnothing$ and $U^{\prime} \times V^{\prime} \cap T\left(U \times V \cap R_{\phi \psi}\right) \neq \varnothing$.

(2.6) LEMMA. Consider diagram 2.2. Suppose $\psi$ is a RIM extension, $Y$ has a dense subset of supprim points and $\phi$ and $\psi$ satisfy one of the conditions in 2.3. Then every non-empty (basic) open set $U \times V \cap R_{\phi \psi}$ contains a point $(x, y)$ such that

$$
E_{\phi}[x] \times\{y\} \subseteq \overline{T\left(U \times V \cap R_{\phi \psi}\right)} .
$$

Proof. By 2.3 and 2.1a, we may assume that $\phi[U]=\psi[V]$. As the supprim points are dense in $Y$, there is a section $\lambda$ for $\phi$ and a $y \in V \cap \operatorname{supp} \lambda_{\psi(y)}$. Let $x \in U$ be such that $\phi(x)=\psi(y)$. Then, $\mathrm{b} ; 1.2$,

$$
E_{\phi}[x] \times\{y\} \subseteq E_{\phi}[x] \times\left(V \cap \operatorname{supp} \lambda_{\psi(y)}\right) \subseteq \overline{T\left(\{x\} \times V \cap R_{\phi \psi}\right)} \subseteq \overline{T\left(U \times V \cap R_{\phi \psi}\right)} .
$$

(2.7) Lemma. Consider diagram 2.2. Suppose $\phi$ is a RIC extension and let $(x, y)$ be an almost periodic point. If $U \times V \cap R_{\phi \psi}$ is a basic open neighbourhood of $(x, y)$ in $R_{\phi \psi}$, then

$$
E_{\phi}[x] \times\{y\} \subseteq \overline{T\left(U \times V \cap R_{\phi \psi}\right)} .
$$

Proof. Let $v \in J$ be such that $v(x, y)=(x, y)$. By 1.10 , there is an open set $W=W(v)$ in $T$ such that $W y \subseteq V$. Define $U:=[U, W] \cap v \phi^{\leftarrow} \psi(x)$. Then $U$ is an $\mathfrak{F}(\mathscr{X}, v)-$ neighbourhood of $x$ in $c \phi^{\leftarrow} \phi(x)$. Let $x^{\prime} \in U$, then $x^{\prime} \in t^{-1} U$ for some $t \in W$, so

$$
\left(x^{\prime}, y\right) \in t^{-1}\left(U \times t y \cap R_{\phi \psi}\right) \subseteq t^{-1}\left(U \times W y \cap R_{\phi \psi}\right) \subseteq T\left(U \times V \cap R_{\phi \psi}\right) .
$$

Consequently, $U \times\{y\} \subseteq T\left(U \times V \cap R_{\phi \psi}\right)$ and so, by $1.16 \mathrm{a}$,

$$
E_{\phi}[x] \times\{y\} \subseteq v \circ(U \times\{y\}) \subseteq \overline{T\left(U \times V \cap R_{\phi \psi}\right)} .
$$

(2.8) Theorem. Consider diagram 2.2. In each of the following cases we have $\phi-\psi$ iff $\theta-\psi$ :

(a) $\phi$ is a RIC extension, $\phi$ and $\psi$ satisfy $\mathrm{gBc}$ (or equivalently, $Y$ has a dense subset of almost periodic points);

(b) $\psi$ is a RIM extension, $Y$ has a dense subset of supprim points and one of the conditions in 2.3 is satisfied.

Proof. (a) Follows from 2.7, 2.5 and 2.4.

(b) Follows from 2.6, 2.5 and 2.4 .

(2.9) In order to prove 2.11 , the $\mathrm{Bc}$ version of 2.8 (thus generalizing $[15,2.6 .3]$ ), we consider a commutative 'double' diagram similar to the one constructed by Veech in [15], as follows:

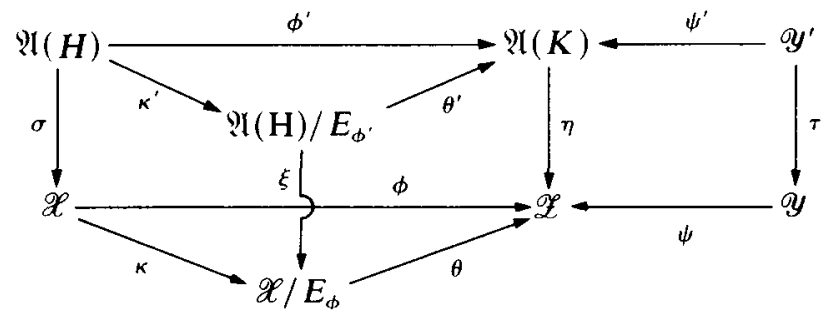


Here $H:=\mathscr{G}\left(\mathscr{X}, x_{0}\right)$ and $K:=\mathscr{G}\left(\mathscr{Z}, z_{0}\right)$ are the Ellis groups of $\mathscr{X}$ and $\mathscr{Z}$ with respect to $x_{0}=u x_{0}$ and $z_{0}=u z_{0}, \phi^{\prime}: \mathfrak{A}(H) \rightarrow \mathfrak{U}(K)$ is the RIC extension defined by $p^{\circ} H \mapsto$ $p \circ K, \sigma$ and $\eta$ are the maximally proximal extensions of $\mathscr{X}$ and $\mathscr{Z}$ (1.13). Then the $\operatorname{ttg} Y^{\prime}$ is a subttg of $y \times \mathfrak{U}(K)$ defined by

$$
Y^{\prime}=\left\{(y, p \circ K) \mid y \in p \circ u \psi^{\leftarrow}\left(x_{0}\right)\right\}
$$

and the maps $\psi^{\prime}: \mathscr{Y}^{\prime} \rightarrow \mathfrak{H}(K)$ and $\tau: \mathscr{Y}^{\prime} \rightarrow \mathscr{Y}$ are just the projections. The following facts are easily verified:

(i) $Y^{\prime}$ is $T$-invariant and closed in $Y \times \mathfrak{A}(K)$;

(ii) $Y^{\prime}$ has a dense subset of almost periodic points;

(iii) $\tau$ : $\mathscr{Y}^{\prime} \rightarrow \mathscr{Y}$ is proximal and $\tau$ is a surjection iff $Y$ has a dense subset of almost periodic points.

As $\sigma$ and $\eta$ are proximal and $\phi$ and $\phi^{\prime}$ are Bc extensions, it follows from 1.14 (remark between parenthesis) that $\xi$ is proximal.

We shall need the following lemma about lifting of ergodicity.

(2.10) LemMA. Let $\phi: \mathscr{X} \rightarrow \mathscr{Y}$ be a surjective proximal homomorphism of ttgs and let $\mathscr{Z}$ have a dense subset of almost periodic points. Then $\mathscr{X}$ is ergodic iff $\mathscr{Y}$ is ergodic.

Proof. Clearly, if $\mathscr{X}$ is ergodic then $\mathscr{Y}$ is ergodic.

Conversely, suppose that $\mathscr{Y}$ is ergodic. Let $A \subseteq X$ with $A=\overline{T A}$ and $A^{\circ} \neq \varnothing$ and let $B:=\overline{X \backslash A}$. Then $B=\overline{T B}$ and $X=A \cup B$. As

$$
\phi[A] \cup \phi[B]=\phi[X]=Y,
$$

$\phi[A]$ or $\phi[B]$ must have a non-empty interior in $Y$, and so, by ergodicity of $\mathscr{Y}$, $\phi[A]=Y$ or $\phi[B]=Y$.

Suppose that $\phi[A]=Y$. Let $x \in X$ be an almost periodic point. Then for some $a \in A, \phi(a)=\phi(x)$. As $\phi$ is proximal, $a$ and $x$ are proximal and by almost periodicity of $x$ we have that $x \in \overline{T a} \subseteq \overline{T A}=A$. Consequently, every almost periodic point in $\mathscr{X}$ is in $A$, so $X=A$. Suppose that $\phi[B]=Y$, then similarly it follows that $X=B$, which contradicts the assumption of $A^{\circ} \neq \varnothing$. Hence $X=A$ and $\mathscr{X}$ is ergodic.

(2.11) TheOREM. Consider diagram 2.2 with $\phi$ a $\mathrm{Bc}$ extension and $\phi$ and $\psi$ satisfying gBc. Then $\phi-\psi$ iff $\theta-\psi$.

Proof. Construct the diagram in 2.9 and suppose that $\theta-\psi$. As $\theta^{\prime}$ is a RIC extension (1.13) and as $\mathscr{Y}^{\prime}$ has a dense subset of almost periodic points, $R_{\theta^{\prime} \psi^{\prime}}$ has a dense subset of almost periodic points (1.9). By 2.10, it follows from the proximality of $\xi \times \tau$ that $R_{\theta^{\prime} \psi^{\prime}}$ is ergodic, so $\theta^{\prime}-\psi^{\prime}$. As $\phi^{\prime}$ is a RIC extension (1.13) and as $\mathscr{Y}^{\prime}$ has a dense subset of almost periodic points, $\phi^{\prime}$ and $\psi^{\prime}$ satisfy $\mathrm{gBc}(1.9)$. By $2.8 \mathrm{a}, \phi^{\prime}-\psi^{\prime}$. Since $\sigma \times \tau\left[R_{\phi^{\prime} \psi^{\prime}}\right]=R_{\phi \psi}$ it follows that $\phi \dot{-\psi}$.

A homomorphism of ttgs $\phi: \mathscr{X} \rightarrow \mathscr{Z}$ is called $n$-weakly mixing iff $R_{\phi}^{n}$ is ergodic. If $\phi$ is 2-weakly mixing then $\phi$ is just called weakly mixing. If $\phi$ is $n$-weakly mixing for every $n \in \mathbb{N}$, then $\phi$ is called totally weakly mixing.

(2.12) THEOREM. Let $\phi: \mathscr{X} \rightarrow \mathscr{Z}$ be a homomorphism of minimal ttgs with $E_{\phi}=R_{\phi}$.

(a) If $\phi$ is $n$-Bc then $\phi$ is $n$-weakly mixing. In particular, if $\phi$ is $n$ - $\mathrm{Bc}$ for every $n \in \mathbb{N}$ (e.g. $\phi$ is a RIC extension) then $\phi$ is totally weakly mixing. 
(b) If $\phi$ is an open RIM extension then $\phi$ is totally weakly mixing.

Proof. Consider diagram 2.2 and note that $E_{\phi}=R_{\phi}$ implies that $\theta$ is an isomorphism. So, under the conditions in 2.8 and 2.11 , we have $\phi-\psi$ iff $\mathscr{Y}$ is ergodic. We shall prove the statements by induction.

(a) First note that $R_{\phi}^{2}=R_{\phi}$ is ergodic (apply 2.11 to $\phi$ and $\phi$ ). Assume $R_{\phi}^{m}$ is ergodic for certain $m$ with $2 \leq m<n$. Then define $\psi: R_{\phi}^{m} \rightarrow Z$ as a restriction of $\phi^{m}$. By 2.11 and the observation that $R_{\theta \psi} \cong R_{\phi}^{m}$, it follows that $R_{\phi \psi}$ is ergodic. So, as $R_{\phi}^{m+1} \cong R_{\phi \psi}, R_{\phi}^{m+1}$ is ergodic.

(b) As $\mathscr{X}$ is minimal, the supprim points are dense in $X$, so $R_{\phi}$ is ergodic (apply to $2.8 \mathrm{~b}$ to $\phi$ and $\phi$ ). Define $\psi: R_{\phi}^{m} \rightarrow Z$ as a restriction of $\phi^{m}$. Then $\lambda^{m}$ is a section for $\psi$ ( $\lambda$ a section for $\phi)$. As $\phi$ is open, one sees readily that $\psi$ is open and that the supprim points are dense in $R_{\phi}^{m}$. Suppose $R_{\phi}^{m}$ is ergodic, then application of $2.8 \mathrm{~b}$ to $\phi$ and $\psi$ shows ergodicity of $R_{\phi}^{m+1}$.

(2.13) .We shall now turn to a generalization of $[14,6.11]$ and $[11,1.9]$. Consider the following diagram of homomorphisms of minimal ttgs:

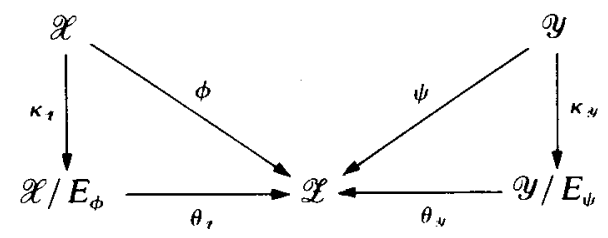

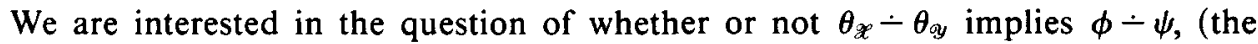
converse is obviously true). First we shall show that $\theta_{\mathscr{X}}-\theta_{\mathscr{Y}}$ iff $\theta_{\mathscr{X}} \perp \theta_{\mathscr{Y}}$.

(2.14) Lemma. Let $\phi: \mathscr{X} \rightarrow \mathscr{Y}$ be a surjective homomorphism of ttgs. Let $X^{\prime} \subseteq X$ be a closed invariant subset of $X$ such that:

(i) $\phi\left[X^{\prime}\right]=Y$;

(ii) $\left.\phi\right|_{X}: X^{\prime} \rightarrow Y$ is open.

If $\mathscr{X}$ is ergodic then $X=Q_{\phi}\left[X^{\prime}\right]$.

Proof. Let $x \in X$ and let $x^{\prime} \in X^{\prime}$ be such that $\phi\left(x^{\prime}\right)=\phi(x)$. As $\mathscr{X}$ is ergodic it follows that $x^{\prime} \in \overline{T \alpha(x)}$ for every $\alpha \in \mathcal{U}_{X}$; so for every $\alpha \in U_{X}$ we have $\alpha\left(x^{\prime}\right) \cap T \alpha(x) \neq \varnothing$. For $\alpha \in U_{X}$ let $x_{\alpha} \in \alpha(x)$ and $t_{\alpha} \in T$ be such that $t_{\alpha} x_{\alpha} \in \alpha\left(x^{\prime}\right)$. Then $x_{\alpha} \rightarrow x$ and $t_{\alpha} x_{\alpha} \rightarrow x^{\prime}$; so $t_{\alpha} \phi\left(x_{\alpha}\right) \rightarrow \phi\left(x^{\prime}\right)$. As $\left.\phi\right|_{X^{\prime}}$ is open, there are $x_{\alpha}^{\prime} \in X^{\prime}$ with $\phi\left(x_{\alpha}^{\prime}\right)=\phi\left(x_{\alpha}\right)$ such that $t_{\alpha} x_{\alpha}^{\prime} \rightarrow x^{\prime}$. For a suitable subnet let $z=\lim x_{\alpha}^{\prime}$. Then $z \in X^{\prime}$ and $(x, z) \in Q_{\phi}$. Hence $x \in Q_{\phi}[z]$ and so $X \subseteq Q_{\phi}\left[X^{\prime}\right]$.

(2.15) Theorem. Let $\phi: \mathscr{X} \rightarrow \mathscr{Y}$ be an almost periodic extension with $\mathscr{X}$ ergodic and $\mathscr{Y}$ minimal. Then $\mathscr{X}$ is minimal.

Proof. Let $X^{\prime}$ be a minimal subset of $X$. As $\left.\phi\right|_{X^{\prime}}$ is almost periodic, $\left.\phi\right|_{X^{\prime}}$ is open. From 2.14 it follows that $X=Q_{\phi}\left[X^{\prime}\right]$. As $\phi$ is almost periodic, $Q_{\phi}=\Delta_{X}$, so $X=X^{\prime}$.

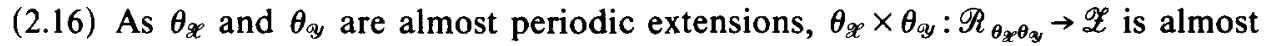
periodic too. By minimality of $\mathscr{Z}$ and 2.15 , it follows that $\theta_{\mathscr{Z}}-\theta_{\mathscr{Y}}$ implies $\theta_{\mathscr{X}} \perp \theta_{\mathscr{Y}}$. 
The other way around is trival. Note that with little extra effort ([1]) one can prove the following:

THEOREM. Let $\phi$ and $\psi$ be HPI extensions of minimal ttgs such that $\phi$ and $\psi$ satisfy $\mathrm{gBc}$, then $\phi-\psi$ iff $\phi \perp \psi$. In particular this holds for distal maps $\phi$ and $\psi$.

(2.17) ThEOREM. Consider the diagram in 2.13. In each of the following cases we have $\phi-\psi$ iff $\theta_{\mathscr{P}}-\theta_{\mathscr{y}}$ iff $\theta_{\mathscr{X}} \perp \theta_{\mathscr{y}}$.

(a) $\phi$ is a $\mathrm{Bc}$ extension and $\phi$ and $\psi$ satisfy $\mathrm{gBc}$;

(b) $\phi$ is a RIM extension and $\phi$ and $\psi$ satisfy $\mathrm{gBc}$

(c) $\phi$ is a RIM extension and $\phi$ or $\psi$ is open.

Proof. Consider diagram 2.13. As almost periodic extensions of minimal ttgs are open RIM extensions, it follows from $2.8 \mathrm{~b}$ that $\theta_{\mathscr{X}}-\psi$ iff $\theta_{\mathscr{X}}-\theta_{\mathscr{X}}$ iff $\phi-\theta_{\mathscr{y}}$.

(a) Assume $\theta_{\mathscr{P}}-\theta_{\text {oyy }}$. Then, by the above, $\theta_{\mathscr{X}}-\psi$. Hence, by $2.11, \phi-\psi$.

(b) and (c) Assume $\theta_{\mathscr{Z}}-\theta_{0 y}$. Then by the above $\theta_{\mathscr{y}}-\phi$. As $\phi$ and $\psi$ satisfy one of the conditions in 2.3 , it follows that $\psi-\phi$ (apply $2.8 \mathrm{~b}$ with $\phi$ and $\psi$ interchanged.)

(2.18) Corollary. Let $\phi: \mathscr{X} \rightarrow \mathscr{L}$ be a homomorphism of minimal ttgs with $E_{\phi}=R_{\phi}$. If $\phi$ is a RIC extension or an open RIM extension then $\phi$ is weakly disjoint from every homomorphism $\psi: \mathscr{Y} \rightarrow \mathscr{Z}$ of minimal ttgs.

Proof. As $E_{\phi}=R_{\phi}$ implies $\theta$ is an isomorphism, this follows immediately from $2.8 \mathrm{a}$ or $2.17 \mathrm{c}$.

\section{A variation on regional proximality}

In studying the equicontinuous structure of a ttg, the notion of the regionally proximal relation is fundamental. It expresses how far the ttg is from being equicontinuous ((uniform) almost periodic). For a deeper understanding of the bad behaviour of certain points that keep the ttg from being equicontinuous, we need a more detailed knowledge of how the regionally proximal relation is produced and why transitivity occurs in the standard cases in which it does.

Let us recall that the regional proximal relation $Q_{\mathscr{X}}$ is the set of points in $X \times X$, such that there exist nets $\left(x_{i}, y_{i}\right) \rightarrow(x, y)$ in $X \times X$ and $\left\{t_{i}\right\}_{i}$ in $T$ such that $t_{i}\left(x_{i}, y_{i}\right) \rightarrow$ $(z, z)$ for some $z \in X$. Here all that is required is the existence of such a net $\left\{\left(x_{i}, y_{i}\right)\right\}_{i}$ without regard to the way it approaches $(x, y)$. Thus there might be 'few' such nets for one pair in $Q_{x}$ and 'many' for another pair, which results in a difference in dynamical behaviour of the pairs.

Now with the above discussion in mind, the meaning of the following notion is clear. First we give a rough description. Let $x$ and $y$ be elements of the ttg $\mathscr{X}$; we say that $(x, y)$ is a sharply regionally proximal pair iff for every net $\left\{\left(x_{i}, y_{i}\right)\right\}_{i}$ tending to $(x, y)$ there is a net $\left\{\left(x_{i}^{\prime}, y_{i}^{\prime}\right)\right\}_{i}$ which is suitably close to the original net but which has the property that there are $t_{i} \in T$ such that $t_{i}\left(x_{i}^{\prime}, y_{i}^{\prime}\right) \rightarrow(z, z)$ for some $z \in X$. Therefore, the net $\left\{\left(x_{i}, y_{i}\right)\right\}_{i}$ provides a direction and $\left\{\left(x_{i}^{\prime}, y_{i}^{\prime}\right)\right\}_{i}$ is the one that makes the pair $(x, y)$ regionally proximal and that follows the direction if it is suitably 
close. Clearly, this notion has a relativized version, that is defined for a homomorphism $\phi: \mathscr{L} \rightarrow \mathscr{Y}$ of ttgs. Now the rigorous definition follows.

Let $\phi: \mathscr{X} \rightarrow \mathscr{Y}$ be a homomorphism of ttgs. We say that $\left(x_{1}, x_{2}\right) \in R_{\phi}$ is a sharply regionally proximal pair iff given some net $\left\{\left(x_{1}^{i}, x_{2}^{i}\right)\right\}_{i}$ in $R_{\phi}$ converging to $\left(x_{1}, x_{2}\right)$ and given neighbourhoods $U^{i}$ of $\left(x_{1}^{i}, x_{2}^{i}\right)$ in $R_{\phi}$, there exist (after passing to suitable subnets) $t_{i}$ in $T$ and $\left(\bar{x}_{1}^{i}, \bar{x}_{2}^{i}\right) \in U^{i}$ such that $t_{i}\left(\bar{x}_{1}^{i}, \bar{x}_{2}^{i}\right) \rightarrow(z, z)$ for some $z \in X$. Denote the collection of sharply regionally proximal pairs for $\phi$ by $Q_{\phi}^{*}$.

The following remark is another way to formulate the notion of sharp regional proximality. The proof is straightforward, thus omitted.

(3.1) Remark. Let $\phi: \mathscr{X} \rightarrow \mathscr{Y}$ be a homomorphism of ttgs. Then

$$
Q_{\phi}^{\#}=\bigcap\left\{\text { int }_{R_{\phi}}\left(\overline{T \alpha \cap R_{\phi}}\right) \mid \alpha \in \mathcal{U}_{X}\right\} \text {. }
$$

(3.2) Examples. Let $\phi: \mathscr{X} \rightarrow \mathscr{Y}$ be a homomorphism of ttgs.

(a) $P_{\phi} \subseteq Q_{\phi}^{\#} \subseteq Q_{\phi}$; so if $\phi$ is proximal $R_{\phi}=P_{\phi}=Q_{\phi}^{\#}=Q_{\phi}=E_{\phi}$.

(b) If $\phi$ is weakly mixing then $R_{\phi}=Q_{\phi}^{\#}=Q_{\phi}=E_{\phi}$.

(c) If $\phi$ is almost periodic then $\Delta_{X}=E_{\phi}=Q_{\phi}=Q_{\phi}^{\#}=P_{\phi}$.

The following example shows that there are minimal ttgs for which $Q \neq Q^{\#}$. Moreover, it shows that if $\phi$ and $\psi$ are homomorphisms of minimal ttgs with $Q_{\phi}=Q_{\phi}^{*}$ and $Q_{\psi}=Q_{\psi}^{*}$ then $Q_{\psi^{\circ} \phi}$ and $Q_{\psi \circ \phi}^{*}$ may be different from each other.

(3.3) EXAMPLE. Let $Y$ be the fourfold covering of the minimal proximal rotation. Then

$$
Q_{\mathscr{y}}^{\#} \neq Q_{\mathscr{y}} \neq E_{\mathscr{y}} \text {. }
$$

Proof. Let $T$ be the free group on two generators. Let $X$ be the circle, define $a: X \rightarrow X$ by $a(x)=x+\alpha$ ( $\alpha$ irrational) and define $b: X \rightarrow X$ by $b(x)=x^{2}$. Then $a$ and $b$ are homeomorphisms of $X$, and $\mathscr{X}$ is a minimal proximal ttg for $T(a, b)$, the minimal proximal rotation. Let $Y$ be the circle and define the map $c: Y \rightarrow Y$ by

$$
c(y):=y+\frac{1}{4} \alpha
$$

and $d: Y \rightarrow Y$ by

$$
d(y):=\frac{1}{4} k+4\left(y-\frac{1}{4} k\right)^{2}
$$

whenever $k \leq 4 y<k+1(k \in\{0,1,2,3\})$. Define the ttg $\mathscr{Y}:=\langle T(c, d), Y\rangle$ and let $\phi: \mathscr{Y} \rightarrow \mathscr{X}$ be defined as $\phi(y)=4 y(\bmod 1)$. Then $\mathscr{Y}($ or better $\phi)$ is the fourfold covering of $\mathscr{X}$.

Note that $P_{\mathscr{Z}}=Q_{\mathscr{Z}}^{\#}=Q_{\mathscr{X}}=E_{\mathscr{Z}}=X \times X$; and that $\phi$ is almost periodic, so that $P_{\phi}=Q_{\phi}^{\#}=Q_{\phi}=E_{\phi}=\Delta_{Y}$.

Obviously, 9 does not admit non-trivial almost periodic factors, in other words $E_{y}=Y \times Y$. As $c$ preserves distances, it is not difficult to see that $\left(y, y^{\prime}\right) \in Q_{y}$ iff the distance $(\bmod 1)$ between $y$ and $y^{\prime}$ is smaller than or equal to $\frac{1}{4}$. So $Q_{y} \neq E_{0 y}$.

If the distance between $y$ and $y^{\prime}$ equals $\frac{1}{4}$, then we can approach $\left(y, y^{\prime}\right)$ with pairs with a distance greater than $\frac{1}{4}$ (from the outside), which shows that $\left(y, y^{\prime}\right) \not \notin Q_{o y}^{*}$. So $Q_{o y} \neq Q_{y}^{\#}$.

An indication of the power of sharp regional proximality is given in the following theorem, which hints at regional proximality of second order which will be discussed in $\S 4$. 
(3.4) THEOREM. Let $\phi: \mathscr{X} \rightarrow \mathscr{Y}$ be a homomorphism of minimal ttgs.

(a) Let $\left(x_{1}, x_{2}\right) \in R_{\phi}$. If $\overline{T\left(x_{1}, x_{2}\right)} \cap Q_{\phi}^{\#} \neq \varnothing$ then we have $\left(x_{1}, x_{2}\right) \in Q_{\phi}^{*}$, and so

$$
\overline{T\left(x_{1}, x_{2}\right)} \subseteq \overline{Q_{\phi}^{\#}} \subseteq Q_{\phi} \text {. }
$$

In particular, if $Q_{\phi}=Q_{\phi}^{\#}$ then $Q_{\phi}$ contains the orbit closures that have a non-empty intersection with $Q_{\phi}$.

(b) Let $\left(x_{1}, x_{2}\right) \in Q_{\phi}^{\#}$ and let $\left\{\left(x_{1}^{i}, x_{2}^{i}\right)\right\}_{i}$ be a net in $R_{\phi}$ converging to $\left(x_{1}, x_{2}\right)$. Choose $\left\{t_{i}\right\}_{i}$ in $T$ and (for a suitable subnet) let $\left(z_{1}, z_{2}\right)=\lim t_{i}\left(x_{1}^{i}, x_{2}^{i}\right)$. Then $\left(z_{1}, z_{2}\right) \in Q_{\phi}$.

(c) If J. $Q_{\phi}^{\#} \subseteq Q_{\phi}^{\#}$ (e.g. $Q_{\phi}^{\#}$ is closed, in particular if $Q_{\phi}=Q_{\phi}^{\#}$ ) then

$$
Q_{\phi}^{*} \circ P_{\phi}=P_{\phi} \circ Q_{\phi}^{*}=Q_{\phi}^{*} \text {. }
$$

Proof. (a) If $\overline{T\left(x_{1}, x_{2}\right)} \cap Q_{\phi}^{\#} \neq \varnothing$ then $\overline{T\left(x_{1}, x_{2}\right)} \cap \operatorname{int}_{R_{\phi}}\left(\overline{T \alpha \cap R_{\phi}}\right) \neq \varnothing$ for every $\alpha \in \mathcal{U}_{X}$, and so

$$
T\left(x_{1}, x_{2}\right) \cap \operatorname{int}_{R_{\phi}}\left(\overline{T \alpha \cap R_{\phi}}\right) \neq \varnothing .
$$

But then it follows that $\left(x_{1}, x_{2}\right) \in$ int $_{R_{\phi}}\left(\overline{T \alpha \cap R_{\phi}}\right)$ for every $\alpha \in \mathcal{U}_{X}$ and consequently $\left(x_{1}, x_{2}\right) \in Q_{\phi}^{\#}$.

(b) Let $\alpha \in U_{X}$. As $\left(x_{1}, x_{2}\right) \in$ int $_{R_{\phi}}\left(\overline{T \alpha \cap R_{\phi}}\right)$, there is an $i(\alpha)$ such that $\left(x_{1}^{i}, x_{2}^{i}\right) \in$ int $_{R_{\phi}}\left(\bar{T} \alpha \cap R_{\phi}\right)$ for every $i \geq i(\alpha)$. But then also $t_{i}\left(x_{1}^{i}, x_{2}^{i}\right) \in$ int $_{R_{\phi}}\left(\overline{T \alpha \cap R_{\phi}}\right)$ for every $i \geq i(\alpha)$ and so

$$
\left(z_{1}, z_{2}\right)=\lim t_{i}\left(x_{1}^{i}, x_{2}^{i}\right) \in \overline{T \alpha \cap R_{\phi}} .
$$

As $\alpha$ was arbitrary it follows that

$$
\left(z_{1}, z_{2}\right) \in \bigcap\left\{\overline{T \alpha \cap R_{\phi}} \mid \alpha \in \mathcal{U}_{X}\right\}=Q_{\phi} .
$$

(c) Let $\left(x_{1}, x_{2}\right) \in P_{\phi}$ and $\left(x_{2}, x_{3}\right) \in Q_{\phi}^{*}$. Let $I$ be a minimal left ideal in $S_{T}$ such that $p x_{1}=p x_{2}$ for every $p \in I$ and let $v \in J_{x_{3}}(I)$. Then

$$
v\left(x_{1}, x_{3}\right)=\left(v x_{1}, x_{3}\right)=\left(v x_{2}, x_{3}\right)=v\left(x_{2}, x_{3}\right) \in J \cdot Q_{\phi}^{*} \subseteq Q_{\phi}^{*} .
$$

By (a), it follows that $\left(x_{1}, x_{3}\right) \in Q_{\phi}^{*}$. Hence $Q_{\phi}^{\#} \circ P_{\phi} \subseteq Q_{\phi}^{*}$. Clearly, $Q_{\phi}^{*} \subseteq Q_{\phi}^{*} \circ P_{\phi}$, so $Q_{\phi}^{*} \circ P_{\phi}=Q_{\phi}^{*}$. In a similar way it follows that $P_{\phi} \circ Q_{\phi}^{*}=Q_{\phi}^{*}$.

Before we can use some results of the preceding section in order to understand the equality $E_{\phi}=Q_{\phi}=Q_{\phi}^{*}$, we need the following lemma.

(3.5) LeMMA. Let $\phi: \mathscr{X} \rightarrow \mathscr{Y}$ be a homomorphism of minimal ttgs and let $\kappa: \mathscr{X} \rightarrow \mathscr{X} / E_{\phi}$ be the quotient map and $\theta: \mathscr{X} / E_{\phi} \rightarrow \mathcal{Y}$ the maximal almost periodic factor of $\phi$. Denote the collection of non-empty open sets in $X / E_{\phi}$ by $O$. Then

$$
E_{\phi}=\bigcap\left\{T\left(\kappa^{\leftarrow}[U] \times \kappa^{+}[U] \cap R_{\phi}\right) \mid U \in O\right\}=\bigcap\left\{\overline{T\left(\kappa^{*}[U] \times \kappa^{t}[U] \cap R_{\phi}\right)} \mid U \in \mathcal{O}\right\} .
$$

Proof. Let $U \in \mathcal{O}$ and $\left(x_{1}, x_{2}\right) \in E_{\phi}$. Then for some $t \in T$ we have $t \kappa\left(x_{1}\right)=t \kappa\left(x_{2}\right) \in U$ and so

$$
\left(x_{1}, x_{2}\right) \in \kappa^{\leftarrow}\left[t^{-1} U\right] \times \kappa^{\leftarrow}\left[t^{-1} U\right] \cap R_{\phi} \subseteq T\left(\kappa^{\leftarrow}[U] \times \kappa^{\leftarrow}[U] \cap R_{\phi}\right) .
$$

Hence

$$
E_{\phi} \subseteq \bigcap\left\{T\left(\kappa^{\leftarrow}[U] \times \kappa^{-}[U] \cap R_{\phi}\right) \mid U \in \mathcal{O}\right\} \subseteq \bigcap\left\{\overline{T\left(\kappa^{-}[U] \times \kappa^{\top}[U] \cap R_{\phi}\right)} \mid U \in \mathcal{O}\right\} .
$$


On the other hand,

$$
\begin{aligned}
& \kappa \times \kappa\left[\cap\left\{\overline{T\left(\kappa^{\leftarrow}[U] \times \kappa^{\leftarrow}[U] \cap R_{\phi}\right)} \mid U \in \mathcal{O}\right\}\right] \\
& \quad \subseteq \bigcap\left\{\overline{T\left(\kappa \times \kappa\left(\kappa-[U] \times \kappa^{\leftarrow}[U] \cap R_{\phi}\right)\right.} \mid U \in \mathcal{O}\right\} \\
& \quad \subseteq \cap\left\{\overline{\left.T(U) \times(U) \cap R_{\theta}\right)} \mid U \in \mathscr{O}\right\}=Q_{\theta}=\Delta_{X / E_{\phi}} .
\end{aligned}
$$

So

$$
\bigcap\left\{\overline{T\left(\kappa^{\leftarrow}[U] \times \kappa^{\leftarrow}[U] \cap R_{\phi}\right)} \mid U \in \mathcal{O}\right\} \subseteq(\kappa \times \kappa)^{\leftarrow}\left[\Delta_{X / E_{\phi}}\right]=E_{\phi}
$$

(3.6) TheOREM. Let $\phi: \mathscr{X} \rightarrow \mathscr{Y}$ be a homomorphism of minimal ttgs and let $\kappa: \mathscr{X} \rightarrow \mathscr{X} / E_{\phi}$ be the quotient map and $\theta: \mathscr{X} / E_{\phi} \rightarrow \mathscr{Y}$ the maximal almost periodic factor of $\phi$. Then the following statements are equivalent:

(a) $E_{\phi}=Q_{\phi}=Q_{\phi}^{\#}$;

(b) for every $\alpha \in \mathcal{U}_{X}$ there is a non-empty open set $V$ in $X$ such that $V=E_{\phi}[V]$ and

$$
V \times V \cap R_{\phi} \subseteq \overline{T \alpha \cap R_{\phi}}
$$

(c) for every open set $U$ in $X$ there is a non-empty open set $V$ in $X$ such that $V=E_{\phi}[V]$ and

$$
V \times V \cap R_{\phi} \subseteq \overline{T\left(U \times U \cap \overline{R_{\phi}}\right)} .
$$

Proof. (b) $\Rightarrow$ (c) As $\mathscr{X}$ is minimal, $T(U \times U)$ is an open set containing the diagonal for every open $U$ in $X$. Hence $\alpha:=T(U \times U) \in U_{X}$.

(c) $\Rightarrow$ (b) For every $\alpha \in \mathcal{U}_{X}$ there is a $\beta \in \mathcal{U}_{X}$ with $\beta=\beta^{-1}$ and $\beta^{2} \subseteq \alpha$. Then

$$
\beta(x) \times \beta(x) \cap R_{\phi} \subseteq \alpha \cap R_{\phi}
$$

for every $x \in X$. Fix $x \in X$ and set $U:=\beta(x)$; then $T\left(U \times U \cap R_{\phi}\right) \subseteq T \alpha \cap R_{\phi}$.

(b) $\Rightarrow$ (a) Let $\alpha \in U_{X}$. By assumption, there is a non-empty open set $V$ in $X$ with $V=E_{\phi}[V]=\kappa^{\leftarrow} \kappa[V]$ and $V \times V \cap R_{\phi} \subseteq \overline{T \alpha \cap R_{\phi}}$. As $\kappa[V]$ is open in $X / E_{\phi}$ it follows from 3.5 that

So

$$
E_{\phi} \subseteq T\left(\kappa^{\leftarrow} \kappa[V] \times \kappa^{\leftarrow} \kappa[V] \cap R_{\phi}\right)=T\left(V \times V \cap R_{\phi}\right)
$$

$$
E_{\phi} \subseteq T\left(V \times V \cap R_{\phi}\right) \subseteq T \cdot \overline{T \alpha \cap R_{\phi}}=\overline{T \alpha \cap R_{\phi}}
$$

and as $T\left(V \times V \cap R_{\phi}\right)$ is an open set in $R_{\phi}, E_{\phi} \subseteq$ int $_{R_{\phi}}\left(\overline{T \alpha \cap R_{\phi}}\right)$. As $\alpha \in \mathcal{U}_{X}$ was arbitrary, it follows that $E_{\phi} \subseteq Q_{\phi}^{*} \subseteq Q_{\phi} \subseteq E_{\phi}$.

(a) $\Rightarrow$ (b) Let $\mathscr{V}$ be the collection of non-empty open sets $V$ in $X$ with $V=E_{\phi}[V]$. Suppose there is an $\alpha \in \mathscr{U}_{X}$ with

$$
V \times V \cap R_{\phi} \cap\left(X \times X \backslash \overline{T \alpha \cap R_{\phi}}\right) \neq \varnothing
$$

for every $V \in \mathscr{V}$. Define

$$
\mathscr{H}(V)=\overline{T\left(V \times V \cap R_{\phi}\right)} \backslash \operatorname{int}_{R_{\phi}}\left(\overline{T \alpha \cap R_{\phi}}\right),
$$

then $\mathscr{K}(V)$ is closed and non-empty for every $V \in \mathscr{V}$. As $\mathscr{V}$ is closed under finite intersections and invariant under $T$, it follows that $\{\mathscr{K}(V) \mid V \in \mathscr{V}\}$ has the finite intersection property. Hence

$$
K:=\bigcap\{\mathscr{K}(V) \mid V \in \mathscr{V}\} \neq \varnothing .
$$

By 3.5, $K \subseteq E_{\phi}$ and by construction $K \cap Q_{\phi}^{\#}=\varnothing$, which contradicts assumption (a). 
(3.7) THEOREM. Let $\mathscr{X}$ and $\mathscr{Y}$ be minimal ttgs. If $\phi: \mathscr{X} \rightarrow \mathscr{Y}$ is an open RIM extension or a $\mathrm{Bc}$ extension then $E_{\phi}=Q_{\phi}=Q_{\phi}^{\#}$.

Proof. First we shall show that $E_{\phi}=Q_{\phi}=Q_{\phi}^{\#}$ if $\phi$ and $\phi$ satisfy the conditions in lemma 2.5. We do this as follows:

Let $U$ be a non-empty open set in $X$. By 2.5 , there is a non-empty open set $\tilde{U}$ with $\tilde{U}=E_{\phi}[\tilde{U}]$ such that $\tilde{U}=\kappa^{*}\left[\kappa[U]^{\circ}\right]($ for $\phi[U]=\phi[U])$ and

$$
\varnothing \neq \tilde{U} \times U \cap R_{\phi} \subseteq \overline{T\left(\bar{U} \times U \cap R_{\phi}\right)} .
$$

Again by 2.5 and by the facts that $\phi[\tilde{U}]=\phi[\tilde{U} \cap U]$ and $\tilde{U}=\kappa^{\leftarrow}\left[\kappa[\tilde{U} \cap U]^{\circ}\right]$ it follows that

$$
\varnothing \neq \tilde{U} \times \tilde{U} \cap R_{\phi} \subseteq \overline{T\left(\tilde{U} \times U \cap R_{\phi}\right)} .
$$

Hence $\tilde{U} \times \tilde{U} \cap R_{\phi} \subseteq \overline{T\left(U \times U \cap R_{\phi}\right)}$ and the theorem follows from 3.6. By 2.6, we know already that an open RIM extension satisfies the conditions in lemma 2.5, which proves the theorem for the open RIM case.

Suppose that $\phi$ is a $\mathrm{Bc}$ extension. Let $U_{1} \times U_{2} \cap R_{\phi}$ be a non-empty (basic) open set in $R_{\phi}$ and let $\left(x_{1}, x_{2}\right) \in U_{1} \times U_{2} \cap R_{\phi}$ be an almost periodic point; say $\left(x_{1}, x_{2}\right)=$ $u\left(x_{1}, x_{2}\right)$ for some $u \in J$. We shall show that

$$
E_{\phi}\left[x_{1}\right] \times\left\{x_{2}\right\} \subseteq \overline{T\left(U_{1} \times U_{2} \cap R_{\phi}\right)} .
$$

Let $V$ be an open set in $T$ with $V=V(u)$ and $V x_{2} \subseteq U_{2}(1.10)$. Define $U:=\left[U_{1}, V\right] \cap$ $u \phi^{\leftarrow} \phi\left(x_{1}\right)$, then $U$ is an $\mathfrak{F}(\mathscr{X}, u)$-neighbourhood of $x_{1}$ in $u \phi^{-} \phi\left(x_{1}\right)$. Consider an arbitrary $x^{\prime} \in U$; say $x^{\prime}=t^{-1} z$ for some $t \in V$ and $z=U_{1}$. Then

$$
\left(x^{\prime}, x_{2}\right)=t^{-1}\left(z, t x_{2}\right) \in T\left(U_{1} \times U_{2}\right),
$$

so $\left(x^{\prime}, x_{2}\right) \in T\left(U_{1} \times U_{2} \cap R_{\phi}\right)$. Hence

$$
U \times\left\{x_{2}\right\} \subseteq T\left(U_{1} \times U_{2} \cap R_{\phi}\right) .
$$

By $1.16 \mathrm{~b}, E_{\phi}\left[x_{1}\right] \subseteq J_{x_{2}} \circ U$, so

$$
E_{\phi}\left[x_{1}\right] \times\left\{x_{2}\right\} \subseteq J_{x_{2}} \circ U \times\left\{x_{2}\right\}=J_{x_{2}} \circ\left(U \times\left\{x_{2}\right\}\right) \subseteq \overline{T\left(U_{1} \times U_{2} \cap R_{\phi}\right)} .
$$

Therefore $\phi$ and $\phi$ satisfy the conditions in lemma 2.5. This proves the Bc case.

(3.8) Corollary. For an amenable group $T$ and any minimal ttg $\mathscr{X}$ for $T$ we have $E_{\mathscr{R}}=Q_{\mathscr{X}}=Q_{\mathscr{Z}}^{\#}$.

The truth of 3.7 is the consequence of certain incompressibilities. As those incompressibilities are preserved under factors, it is natural to ask whether the property $E_{\phi}=Q_{\phi}=Q_{\phi}^{\#}$ is preserved under factors too. To that end we consider the following diagram of homomorphisms of minimal ttgs:

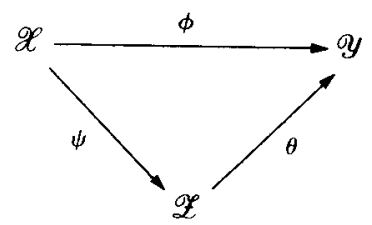


(3.9) Theorem. Consider the diagram following 3.8. If $\psi$ is open then $Q_{\phi}=Q_{\phi}^{\#}$ implies $Q_{\theta}=Q_{\theta}^{*}$. In particular, if $\psi$ is open then $E_{\phi}=Q_{\phi}=Q_{\phi}^{*}$ implies $E_{\theta}=Q_{\theta}=Q_{\theta}^{*}$.

Proof. If $\psi$ is open then $\psi \times\left.\psi\right|_{R_{\phi}}: R_{\phi} \rightarrow R_{\theta}$ is an open homomorphism of ttgs, since $\psi \times \psi: X \times X \rightarrow Z \times Z$ is open and $R_{\phi}=(\psi \times \psi)^{-}\left[R_{\theta}\right]$. Let $\alpha \in \mathcal{U}_{Z}$; then there is a $\beta \in \mathcal{U}_{X}$ such that $\psi \times \psi[\beta] \subseteq \alpha$, hence

$$
\overline{T \cdot \psi \times \psi\left[\beta \cap R_{\phi}\right]} \subseteq \overline{T \alpha \cap R_{\theta}} .
$$

Since $Q_{\theta}=\psi \times \psi\left[Q_{\phi}\right]\left([12,3.2)\right.$, we have, assuming that $Q_{\phi}=Q_{\phi}^{\#}$

$$
Q_{\theta}=\psi \times \psi\left[Q_{\phi}^{\#}\right] \subseteq \psi \times \psi\left[\operatorname{int}_{R_{\phi}}\left(\overline{T \beta \cap R_{\phi}}\right)\right] .
$$

As $\psi \times\left.\psi\right|_{R_{\phi}}$ is open

$$
\left.Q_{\theta} \subseteq \operatorname{int}_{R_{\theta}}\left(\psi \times \psi\left[\overline{T \beta \cap R_{\phi}}\right]\right)=\operatorname{int}_{R_{\theta}}\left(\overline{T \psi \times \psi\left[\beta \cap R_{\phi}\right.}\right]\right) .
$$

Hence it follows that

$$
Q_{\theta} \subseteq \operatorname{int}_{R_{\theta}}\left(\overline{T \psi \times \psi\left[\beta \cap R_{\phi}\right]}\right) \subseteq \operatorname{int}_{R_{\theta}}\left(\overline{T \alpha \cap R_{\theta}}\right) .
$$

As $\alpha \in \mathcal{U}_{z}$ was arbitrary, it follows that $Q_{\theta} \subseteq Q_{\theta}^{\#}$; so $Q_{\theta}=Q_{\theta}^{*}$. (In particular, if $E_{\phi}=Q_{\phi}$ then, by $[12,3.2,3.3]$, it follows that $E_{\theta}=Q_{\theta}$ )

(3.10) TheOREM. Consider the diagram following 3.8. If $Q_{\phi}=(\psi \times \psi)^{\leftarrow}\left[Q_{\theta}\right]$ then $Q_{\phi}=Q_{\phi}^{*}$ implies $Q_{\theta}=Q_{\theta}^{*}$.

Proof. Let $\beta \in \mathscr{U}_{Z}$ and let $\alpha \in \mathcal{U}_{X}$ be such that $\psi \times \psi[\alpha] \subseteq \beta$. Then

$$
\psi \times \psi\left[\overline{T \alpha \cap R_{\phi}}\right] \subseteq \overline{T \psi \times \psi[\alpha] \cap R_{\theta}} \subseteq \overline{T \beta \cap R_{\theta}} .
$$

Suppose $Q_{\phi}=Q_{\phi}^{\#}$ then

$$
Q_{\phi} \subseteq \text { int }_{R_{\phi}}\left(\overline{T \alpha \cap R_{\phi}}\right)=R_{\phi} \backslash \operatorname{cl}_{R_{\phi}}\left(R_{\phi} \backslash\left(\overline{T \alpha \cap R_{\phi}}\right)\right) .
$$

As $Q_{\phi}=(\psi \times \psi)^{-} Q_{\theta}=(\psi \times \psi)^{-}(\psi \times \psi)\left[Q_{\phi}\right]$ it follows that

$$
\begin{aligned}
Q_{\theta} & =\psi \times \psi\left[Q_{\phi}\right] \subseteq \psi \times \psi\left[R_{\phi}\right] \backslash \dot{\psi} \times \psi\left[\mathrm{cl}_{R_{\phi}}\left(R_{\phi} \backslash\left(\overline{T \alpha \cap R_{\phi}}\right)\right)\right] \\
& \subseteq R_{\theta} \backslash \mathrm{cl}_{R_{\theta}}\left(R_{\theta} \backslash \psi \times \psi\left[\overline{T \alpha \cap R_{\phi}}\right]\right) \\
& =\text { int }_{R_{\theta}}\left(\psi \times \psi\left[\overline{T \alpha \cap R_{\phi}}\right]\right) \subseteq \operatorname{int}_{R_{\theta}}\left(\overline{T \psi \times \psi[\alpha] \cap R_{\theta}}\right) \subseteq \operatorname{int}_{R_{\theta}}\left(\overline{T \beta \cap \overline{R_{\theta}}}\right) .
\end{aligned}
$$

As $\beta$ was arbitrary this shows that $Q_{\theta} \subseteq Q_{\theta}^{\#}$.

(3.11) Remark. Consider the diagram following 3.8. If $E_{\phi}=Q_{\phi}$ and if $R_{\psi} \subseteq Q_{\phi}$ then $Q_{\phi}=(\psi \times \psi)^{\leftarrow}\left[Q_{\theta}\right]$.

Proof. Note that $\psi \times \psi\left[Q_{\phi}\right]=Q_{\theta}$, hence $Q_{\phi} \subseteq(\psi \times \psi)^{+}\left[Q_{\theta}\right]$. Let $\left(x_{1}, x_{2}\right) \in$ $(\psi \times \psi)^{-}\left[Q_{\theta}\right]$. Then there is a $\left(z_{1}, z_{2}\right) \in Q_{\phi}$ such that $\psi \times \psi\left(z_{1}, z_{2}\right)=\psi \times \psi\left(x_{1}, x_{2}\right)$. But then $\left(x_{1}, z_{1}\right) \in R_{\psi}$ and also $\left(x_{2}, z_{2}\right) \in R_{\psi}$. Hence

$$
\left(x_{1}, x_{2}\right) \in R_{\psi} \circ Q_{\phi} \circ R_{\psi} \subseteq Q_{\phi}^{3},
$$

and so $\left(x_{1}, x_{2}\right) \in E_{\phi}=Q_{\phi}$.

By now we are able to prove that the equality $E_{\phi}=Q_{\phi}=Q_{\phi}^{\#}$ is preserved under factors.

(3.12) Theorem. Consider the diagram following 3.8. If $E_{\phi}=Q_{\phi}=Q_{\phi}^{*}$ then $E_{\theta}=$ $Q_{\theta}=Q_{\theta}^{*}$. 
Proof. Note that $E_{\phi}=Q_{\phi}$ implies that $E_{\theta}=Q_{\theta}$. Now consider the following diagram of homomorphisms of minimal ttgs.

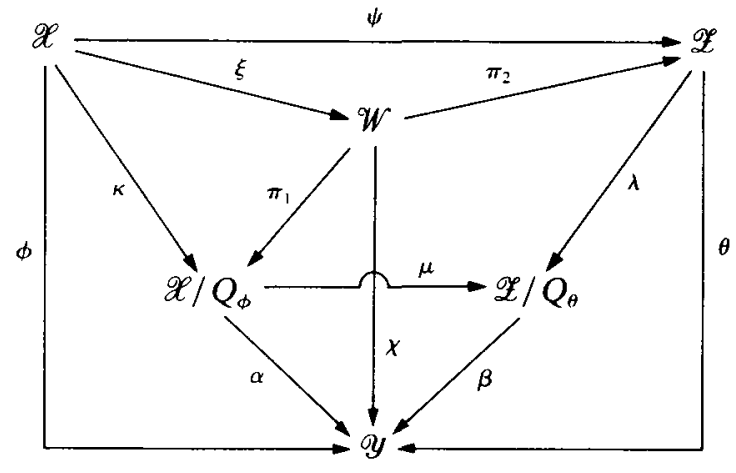

Let $\kappa: \mathscr{X} \rightarrow \mathscr{X} / Q_{\phi}$ and $\lambda: \mathscr{Z} \rightarrow \mathscr{Z} / Q_{\theta}$ be the quotient maps. Since $\psi \times \psi\left[Q_{\phi}\right]=Q_{\theta}$ there exists a unique homomorphism $\mu: \mathscr{X} / Q_{\phi} \rightarrow \mathscr{Y} / Q_{\theta}$ such that $\lambda \circ \psi=\mu \circ \kappa$. As $\alpha=\beta \circ \mu$, $\mu$ is almost periodic. Let $x \in u X, z:=\psi(x)$ and note that $(\kappa(x), z) \in R_{\mu \lambda}$. Define $W:=\overline{T(\kappa(x), z)}$, then $W$ is a minimal subset of $R_{\mu \lambda}$ (for $J_{x} \subseteq J_{\kappa(x)} \cap J_{z}$ ) and $W$ projects onto $X / Q_{\phi}$ and $Z$ by $\pi_{1}$ and $\pi_{2}$ respectively. It is an elementary exercise to show that $\pi_{2}$ is an almost periodic map ( $\mu$ is almost periodic!), so $\pi_{2}$ is open. Define $\chi: \mathscr{W} \rightarrow \mathscr{Y}$ by $\chi=\alpha \circ \pi_{1}$ and let $\xi: \mathscr{X} \rightarrow \mathscr{W}$ be defined by $\xi(x)=(\kappa(x), z)$. Then $\phi=\chi \circ \xi$. As, clearly, $R_{\xi} \subseteq R_{\kappa}=Q_{\phi}$ it follows from 3.11 that $Q_{\phi}=(\xi \times \xi)^{\leftarrow}\left[Q_{\chi}\right]$. Hence by 3.10, we know that $Q_{\chi}=Q_{\chi}^{*}$. As $\chi=\theta \circ \pi_{2}$ and $\pi_{2}$ is open it follows from 3.9 that $Q_{\theta}=Q_{\theta}^{\#}$, which proves the theorem.

In 3.7 we have seen that $E_{\phi}=Q_{\phi}=Q_{\phi}^{\#}$ in the case of open RIM extensions and of Bc extensions. Is the equality of those three relations a coincidence? We shall see that it is not; at least, we shall see that in several situations the equality of $Q_{\phi}$ and $Q_{\phi}^{\#}$ implies $E_{\phi}=Q_{\phi}=Q_{\phi}^{*}$. Whether or not transitivity of $Q_{\phi}$ implies $Q_{\phi}=Q_{\phi}^{*}$ is unknown. First we introduce some notation:

Let $\phi: \mathscr{X} \rightarrow \mathscr{Y}$ be a homomorphism of minimal ttgs. Let $\left(x_{1}, x_{2}\right) \in R_{\phi}$ and $p \in S_{T}$. Then define

$$
p *\left(x_{1}, x_{2}\right):=\bigcap\left\{p \circ V \mid V \text { is a neighbourhood of }\left(x_{1}, x_{2}\right) \text { in } R_{\phi}\right\} .
$$

Clearly, $p *\left(x_{1}, x_{2}\right)=\bigcap\left\{p \circ\left(U_{1} \times U_{2} \cap R_{\phi}\right) \mid U_{i} \in \mathscr{V}_{x_{i}}\right\}$ (we denote the neighbourhood system of $x$ in $X$ by $\mathscr{V}_{x}$ ). Note that there is some ambiguity in the notation as we do not specify the map. As we use it only in the situation of one specific homomorphism $\phi$ and never with respect to $X \times X$, no serious problem will arise, (compare with the definition of $*$ at the beginning of $\S 4$ ).

(3.13) THEOREM. Let $\phi: \mathscr{X} \rightarrow \mathscr{y}$ be a homomorphism of ttgs (not necessarily minimal) and let $\left(x_{1}, x_{2}\right) \in R_{\phi}$. Then $\left(x_{1}, x_{2}\right) \in Q_{\phi}$ iff there is a minimal left ideal I in $S_{T}$ with

$$
p *\left(x_{1}, x_{2}\right) \cap \Delta_{X} \neq \varnothing \quad \text { for every } p \in I \text {. }
$$

Proof. Let $\left(x_{1}, x_{2}\right) \in Q_{\phi}$. Then there are nets $\left\{\left(x_{1}^{i}, x_{2}^{i}\right)\right\}_{i}$ and $\left\{t_{i}\right\}_{i}$ in $R_{\phi}$ and $T$ such that $\left(x_{1}^{i}, x_{2}^{i}\right) \rightarrow\left(x_{1}, x_{2}\right)$ and $t_{i}\left(x_{1}^{i}, x_{2}^{i}\right) \rightarrow(x, x)$ for some $x \in X$. Without loss of generality we may assume that the net $\left\{t_{i}\right\}_{i}$ converges to some $p \in S_{T}$. Let $V$ be a neighbourhood 
of $\left(x_{1}, x_{2}\right)$ in $R_{\phi}$. Then there is an $i_{0}$ such that $\left(x_{1}^{i}, x_{2}^{i}\right) \in V$ for every $i \geq i_{0}$. Hence

$$
(x, x)=\lim \left\{t_{i}\left(x_{1}^{i}, x_{2}^{i}\right) \mid i \geq i_{0}\right\} \in \lim t_{i} \bar{V}=p \circ V .
$$

As $V$ was arbitrary, $(x, x) \in p *\left(x_{1}, x_{2}\right)$ and so $p *\left(x_{1}, x_{2}\right) \cap \Delta_{X} \neq \varnothing$.

Conversely, suppose that for some $p \in S_{T}$ we have $p *\left(x_{1}, x_{2}\right) \cap \Delta_{X} \neq \varnothing$, say $(x, x) \in p *\left(x_{1}, x_{2}\right)$. For $\alpha \in U_{X}$,

$$
p \circ\left(\alpha\left(x_{1}\right) \times \alpha\left(x_{2}\right) \cap R_{\phi}\right) \in 2^{R_{\phi}}
$$

and $\left\langle\left(\alpha \cap R_{\phi}\right)^{\circ}, R_{\phi}\right\rangle$ is a neighbourhood of $p \circ\left(\alpha\left(x_{1}\right) \times \alpha\left(x_{2}\right) \cap R_{\phi}\right)$ in $2^{R_{\phi}}$. Let $\left\{t_{i}\right\}_{i}$ be a net in $T$ with $t_{i} \rightarrow p$ in $S_{T}$. Then

$$
t_{i}\left(\overline{\alpha\left(x_{1}\right) \times \alpha\left(x_{2}\right) \cap R_{\phi}}\right) \rightarrow p \circ\left(\alpha\left(x_{1}\right) \times \alpha\left(x_{2}\right) \cap R_{\phi}\right) \text { in } 2^{R_{\phi}} \text {. }
$$

So there is an $i_{\alpha}$ such that

$$
t_{i_{\alpha}}\left(\overline{\alpha\left(x_{1}\right) \times \alpha\left(x_{2}\right) \cap R_{\phi}}\right) \cap\left(\alpha \cap R_{\phi}\right)^{\circ} \neq \varnothing .
$$

Hence $t_{i_{\alpha}}\left(\alpha\left(x_{1}\right) \times \alpha\left(x_{2}\right) \cap R_{\phi}\right) \cap \alpha \cap R_{\phi} \neq \varnothing$ and we can find $t_{\alpha}:=t_{i_{\alpha}}$ in $T$ and $\left(x_{1}^{\alpha}, x_{2}^{\alpha}\right) \in \alpha\left(x_{1}\right) \times \alpha\left(x_{2}\right) \cap R_{\phi}$ such that $t_{\alpha}\left(x_{1}^{\alpha}, x_{2}^{\alpha}\right) \in \alpha \cap R_{\phi}$. Doing this for every $\alpha \in \mathcal{U}_{X}$, we obtain nets $\left\{t_{\alpha}\right\}_{\alpha \in \mathcal{U}_{X}}$ in $T$ and $\left\{\left(x_{1}^{\alpha}, x_{2}^{\alpha}\right)\right\}_{\alpha \in \mathcal{U}_{X}}$ in $R_{\phi}$ such that

$$
\left(x_{1}^{\alpha}, x_{2}^{\alpha}\right) \rightarrow\left(x_{1}, x_{2}\right) \text { and } t_{\alpha}\left(x_{1}^{\alpha}, x_{2}^{\alpha}\right) \rightarrow(x, x) \text {. }
$$

Consequently, $\left(x_{1}, x_{2}\right) \in Q_{\phi}$. What we have proved by now is

$$
\left(x_{1}, x_{2}\right) \in Q_{\phi} \text { iff } p *\left(x_{1}, x_{2}\right) \cap \Delta_{X} \neq \varnothing \quad \text { for some } p \in S_{T},
$$

hence the 'if' part of the theorem is proved.

Let $\left(x_{1}, x_{2}\right) \in Q_{\phi}$ and define

$$
S:=\left\{p \in S_{T} \mid p *\left(x_{1}, x_{2}\right) \cap \Delta_{X} \neq \varnothing\right\}
$$

By the above, $S \neq \varnothing$ and, clearly, $S$ is $T$-invariant. We shall show that $S$ is closed; hence it follows that $S$ contains a minimal left ideal, which proves the theorem.

For each neighbourhood $V$ of $\left(x_{1}, x_{2}\right)$ in $R_{\phi}$ the mapping $p \mapsto p \circ V$ is continuous, hence the mapping

$$
\Psi: p \mapsto \bigcap\left\{p \circ V \mid V \text { neighbourhood of }\left(x_{1}, x_{2}\right) \text { in } R_{\phi}\right\}: S_{T} \rightarrow 2^{R_{\phi}}
$$

is upper semi continuous. Since $\Delta_{X}$ is closed and as $S$ is the pre-image under $\Psi$ of the closed subset $\left\{A \in 2^{R^{\phi}} \mid A \cap \Delta_{X} \neq \varnothing\right\}$ of $2^{R_{\phi}}$, it follows that $S$ is closed.

The following remark in fact repeats and extends $3.4 \mathrm{a}$ and $\mathrm{b}$.

(3.14) REMARK. Let $\phi: \mathscr{X} \rightarrow \mathscr{Y}$ be a homomorphism of ttgs and let $\left(x_{1}, x_{2}\right) \in R_{\phi}$.

(a) If $\left(x_{1}, x_{2}\right) \in Q_{\phi}^{*}$, then $p *\left(x_{1}, x_{2}\right) \subseteq Q_{\phi}$ for every $p \in S_{T}$.

(b) If $p *\left(x_{1}, x_{2}\right) \cap Q_{\phi}^{\#} \neq \varnothing$ for some $p \in S_{T}$, then $\left(x_{1}, x_{2}\right) \in Q_{\phi}$.

Proof. (a) Let $\alpha \in U_{X}$, then $\left(x_{1}, x_{2}\right) \in$ int $_{R_{\phi}}\left(\overline{T \alpha \cap R_{\phi}}\right)$. So there are open neighbourhoods $U_{1} \in \mathscr{V}_{x_{1}}$ and $U_{2} \in \mathscr{V}_{x_{2}}$ such that

$$
\left(x_{1}, x_{2}\right) \in U_{1} \times U_{2} \cap R_{\phi} \subseteq \text { int }_{R_{\phi}}\left(\overline{T \alpha \cap R_{\phi}}\right) .
$$

For every $p \in S_{T}$ it follows that

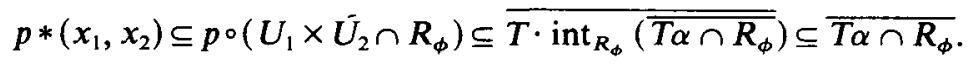

As $\alpha$ was arbitrary, $p *\left(x_{1}, x_{2}\right) \subseteq Q_{\phi}$ for every $p \in S_{T}$.

(b) Suppose $p *\left(x_{1}, x_{2}\right) \cap Q_{\phi}^{*} \neq \varnothing$. Let $\left\{t_{i}\right\}_{i}$ be a net in $T$ with $t_{i} \rightarrow p$ and let 
$\alpha, \beta \in \mathcal{U}_{X}$ be such that $\beta \subseteq \alpha$. Then

$$
p \circ\left(\beta\left(x_{1}\right) \times \beta\left(x_{2}\right) \cap R_{\phi}\right) \cap \operatorname{int}_{R_{\phi}}\left(\overline{T \alpha \cap R_{\phi}}\right) \neq \varnothing
$$

and as $\left\langle\right.$ int $\left._{R_{\phi}}\left(\overline{T \alpha \cap R_{\phi}}\right), R_{\phi}\right\rangle$ is an open neighbourhood of the element $p \circ\left(\beta\left(x_{1}\right) \times\right.$ $\left.\beta\left(x_{2}\right) \cap R_{\phi}\right)$ of $2^{R_{\phi}}$, while

$$
t_{i}\left(\overline{\beta\left(x_{1}\right) \times \beta\left(x_{2}\right) \cap R_{\phi}}\right) \rightarrow p \circ\left(\beta\left(x_{1}\right) \times \beta\left(x_{2}\right) \cap R_{\phi}\right),
$$

it follows that eventually

$$
t_{i}\left(\overline{\beta\left(x_{1}\right) \times \beta\left(x_{2}\right) \cap R_{\phi}}\right) \cap \operatorname{int}_{R_{\phi}}\left(\overline{T \alpha \cap R_{\phi}}\right) \neq \varnothing .
$$

But then $\beta\left(x_{1}\right) \times \beta\left(x_{2}\right) \cap \overline{T \alpha \cap R_{\phi}} \neq \varnothing$, and as is easily seen $\left(x_{1}, x_{2}\right) \in \overline{T \alpha \cap R_{\phi}}$. Consequently, $\left(x_{1}, x_{2}\right) \in Q_{\phi}$.

(3.15) LEMMA. Let $\phi: \mathscr{X} \rightarrow \mathscr{Y}$ be a homomorphism of ttgs and suppose that $Q_{\phi}=Q_{\phi}^{*}$. Let $(x, y) \in Q_{\phi}$ and $(y, z) \in Q_{\phi}$. If $\phi$ is open in $x \in X$, then $(x, z) \in Q_{\phi}$.

Proof. By 3.13, we can find a minimal left ideal $I$ in $S_{T}, p \in I$ and a $z^{\prime} \in X$ such that $\left(z^{\prime}, z^{\prime}\right) \in p *(y, z)$. Let $\alpha \in U_{X}$ and let $U_{x} \subseteq \alpha(x), U_{y} \subseteq \alpha(y)$ and $U_{z} \subseteq \alpha(z)$ be open neighbourhoods of $x, y$ and $z$ in $X$, such that

$$
U_{x} \times U_{y} \cap R_{\phi} \subseteq \operatorname{int}_{R_{\phi}}\left(\overline{T \alpha \cap R_{\phi}}\right)
$$

(no further conditions on $U_{z}$ ). As $\phi$ is open in $x$, we may assume that $U_{y}$ is such that $\phi\left[U_{y}\right] \subseteq \phi\left[U_{x}\right]$. Since

$$
\left(z^{\prime}, z^{\prime}\right) \in p^{*}(y, z) \subseteq p \circ\left(U_{y} \times U_{z} \cap R_{\phi}\right)
$$

we can find nets $\left\{t_{i}\right\}_{i}$ in $T$ and $\left\{\left(y_{i}, z_{i}\right)\right\}_{i}$ in $U_{y} \times U_{z} \cap R_{\phi}$ such that $p=\lim t_{i}$ and $\left(z^{\prime}, z^{\prime}\right)=\lim t_{i}\left(y_{i}, z_{i}\right)$. Let $x_{i} \in U_{x}$ be such that $\phi\left(x_{i}\right)=\phi\left(y_{i}\right)$. Then, for every $i$,

$$
\left(x_{i}, y_{i}\right) \in U_{x} \times U_{y} \cap R_{\phi} \text { and }\left(x_{i}, z_{i}\right) \in U_{x} \times U_{z} \cap R_{\phi} .
$$

Let $x_{\alpha}^{\prime}:=\lim t_{i} x_{i}$ (after passing to a suitable subnet). Then

$$
\left(x_{\alpha}^{\prime}, z^{\prime}\right)=\lim t_{i}\left(x_{i}, y_{i}\right) \in p^{\circ}\left(U_{x} \times U_{y} \cap R_{\phi}\right) \subseteq p^{\circ}\left(\overline{T \alpha \cap R_{\phi}}\right) \subseteq \overline{T \alpha \cap R_{\phi}}
$$

and

$$
\left(x_{\alpha}^{\prime}, z^{\prime}\right)=\lim t_{i}\left(x_{i}, z_{i}\right) \in p \circ\left(U_{x} \times U_{z} \cap R_{\phi}\right) \subseteq p^{\circ}\left(\alpha(x) \times \alpha(z) \cap R_{\phi}\right) .
$$

So for every $\alpha \in \mathcal{U}_{X}$ we can define in this way an element $x_{\alpha}^{\prime} \in X$. Let $x^{\prime}=\lim x_{\beta}^{\prime}$ (after passing to a suitable subnet). Then

$$
\left(x^{\prime}, z^{\prime}\right)=\lim \left(x_{\beta}^{\prime}, z^{\prime}\right) \in \overline{T \alpha \cap R_{\phi}} \quad \text { for every } \alpha \in \mathcal{U}_{X}
$$

hence $\left(x^{\prime}, z^{\prime}\right) \in Q_{\phi}=Q_{\phi}^{*}$. And

$$
\left(x^{\prime}, z^{\prime}\right)=\lim \left(x_{\beta}^{\prime}, z^{\prime}\right) \in p^{\circ}\left(\alpha(x) \times \alpha(z) \cap R_{\phi}\right) \quad \text { for every } \alpha \in u_{X} .
$$

As $p *(x, z)=\bigcap\left\{p \circ\left(\alpha(x) \times \alpha(z) \cap R_{\phi}\right) \mid \alpha \in \mathcal{U}_{X}\right\}$, it follows that $\left(x^{\prime}, z^{\prime}\right) \in p *(x, z)$ and so that $p *(x, z) \cap Q_{\phi}^{*} \neq \varnothing$. By 3.14b, it follows that $(x, z) \in Q_{\phi}$.

(3.16) THEOREM. Let $\phi: \mathscr{X} \rightarrow \mathscr{Y}$ be a homomorphism of minimal ttgs, such that $\phi$ is open in some point $x \in X$. Then $Q_{\phi}=Q_{\phi}^{*}$ implies $E_{\phi}=Q_{\phi}$.

Proof. Let $\left(x_{1}, x_{2}\right) \in Q_{\phi}$ and $\left(x_{2}, x_{3}\right) \in Q_{\phi}$ and let $p \in M$ be such that $x=p x_{1}$. Then $\left(x, p x_{2}\right)=p\left(x_{1}, x_{2}\right) \in Q_{\phi}$ and $\left(p x_{2}, p x_{3}\right) \in Q_{\phi}$; so, by 3.15 , it follows that $\left(x, p x_{3}\right) \in Q_{\phi}$. Let $v \in J_{x_{1}}$, then

$$
\left(x_{1}, v x_{3}\right)=v p^{-1}\left(x, p x_{3}\right) \in Q_{\phi}
$$


As $\left(v x_{3}, x_{3}\right) \in P_{\phi}$ we have $\left(x_{1}, x_{3}\right) \in P_{\phi} \circ Q_{\phi}$. So, by $3.4 \mathrm{c},\left(x_{1}, x_{3}\right) \in Q_{\phi}$. Hence $Q_{\phi} \circ Q_{\phi} \subseteq$ $Q_{\phi}$ and $Q_{\phi}$ is an equivalence relation.

(3.17) COROllary. (a) If $\phi: \mathscr{X} \rightarrow \mathscr{Y}$ is a RIM extension or if $\phi$ is a homomorphism of metric minimal ttgs, then $Q_{\phi}=Q_{\phi}^{*}$ implies $E_{\phi}=Q_{\phi}=Q_{\phi}^{*} ;($ use $[3,3.12 .18])$.

(b) If $\mathscr{X}$ is a minimal ttg then $Q_{\mathscr{X}}=Q_{\mathscr{X}}^{*}$ implies $E_{\mathscr{X}}=Q_{\mathscr{X}}=Q_{\mathscr{X}}^{\#}$.

It is not known whether or not $Q_{\phi}=Q_{\phi}^{*}$ implies $E_{\phi}=Q_{\phi}$ without further restrictions on $\phi$. We shall now give some.other conditions on $\phi$ that are sufficient to deduce $E_{\phi}=Q_{\phi}$ from $Q_{\phi}=Q_{\phi}^{*}$.

(3.18) THEOREM. Consider the diagram following 3.8, and suppose that $\psi$ is proximal. In each of the following two cases we have $Q_{\phi}=Q_{\phi}^{*}$ implies $E_{\phi}=Q_{\phi}=Q_{\phi}^{\#}$.

(a) $\theta$ is open;

(b) $E_{\theta}=Q_{\theta} \circ P_{\theta}$; e.g. $\theta$ is a RIM extension.

Proof. If $\psi$ is proximal, then

$$
(\psi \times \psi)^{\leftarrow}\left[Q_{\theta}\right] \subseteq R_{\psi} \circ Q_{\phi} \circ R_{\psi} \subseteq P_{\phi} \circ Q_{\phi} \circ P_{\phi},
$$

and so, if $Q_{\phi}=Q_{\phi}^{*}$, it follows from $3.4 \mathrm{c}$ that $(\psi \times \psi)^{\leftarrow}\left[Q_{\theta}\right] \subseteq Q_{\phi}$. Hence, by 3.10 , $Q_{\phi}=Q_{\phi}^{*}$ implies $Q_{\theta}=Q_{\theta}^{*}$. But then, in both cases (a) and (b), it follows that $E_{\theta}=Q_{\theta}$ (cf. 3.6 and 3.4 c respectively), As $\psi$ is proximal and as

$$
\psi \times \psi\left[E_{\phi}\right]=E_{\theta}=Q_{\theta}=\psi \times \psi\left[Q_{\phi}\right]
$$

it follows that $E_{\phi} \subseteq P_{\phi} \circ Q_{\phi} \circ P_{\phi}$. But, again by $3.4 \mathrm{c}$, this gives

$$
E_{\phi} \subseteq P_{\phi} \circ Q_{\phi} \circ P_{\phi}=P_{\phi} \circ Q_{\phi}^{*} \circ P_{\phi}=Q_{\phi}^{\#} \text {. }
$$

(3.19) THEOREM. Let $\phi: \mathscr{Z} \rightarrow \mathscr{Y}$ be a homomorphism of minimal ttgs and let $\phi=\theta \circ \psi$. Suppose $\psi$ is open, $R_{\psi} \subseteq Q_{\phi}$ and let $E_{\theta}=Q_{\theta} \circ P_{\theta}$. Then $Q_{\phi}=Q_{\phi}^{*}$ implies $E_{\phi}=Q_{\phi}=Q_{\phi}^{*}$.

Proof. As $\psi$ is open, $Q_{\phi}=Q_{\phi}^{\#}$ implies $Q_{\theta}=Q_{\theta}^{\#}$ by 3.9. Hence, by 3.4c, it follows that

$$
E_{\theta}=Q_{\theta} \circ P_{\theta}=Q_{\theta}^{\#} \circ P_{\theta}=Q_{\theta}^{\#}=Q_{\theta} .
$$

Also, by the openness of $\psi$ we have that $\psi \times \psi: R_{\phi} \rightarrow R_{\theta}$ is an open map. We shall show that $Q_{\phi}=(\psi \times \psi)^{-}\left[Q_{\theta}\right]$, hence that $Q_{\phi}$ is an equivalence relation.

Let $\left(x_{1}, x_{2}\right) \in(\psi \times \psi)^{\leftarrow}\left[Q_{\theta}\right]$; then $\left(z_{1}, z_{2}\right):=\psi \times \psi\left(x_{1}, x_{2}\right) \in Q_{\theta}$. So there are nets $\left\{\left(z_{1}^{i}, z_{2}^{i}\right)\right\}_{i}$ in $R_{\theta}$ and $\left\{t_{i}\right\}_{i}$ in $T$ such that $\left(z_{1}^{i}, z_{2}^{i}\right) \rightarrow\left(z_{1}, z_{2}\right)$ and $t_{i}\left(z_{1}^{i}, z_{2}^{i}\right) \rightarrow\left(z_{1}, z_{1}\right)$. As $\left(x_{1}, x_{2}\right) \in(\psi \times \psi)^{-}\left(z_{1}, z_{2}\right)$ and as the map $\psi \times \psi: R_{\phi} \rightarrow R_{\theta}$ is open, we can find $\left(x_{1}^{i}, x_{2}^{i}\right)$ in $R_{\phi}$ such that $\psi \times \psi\left(x_{1}^{i}, x_{2}^{i}\right)=\left(z_{1}^{i}, z_{2}^{i}\right)$ and $\left(x_{1}^{i}, x_{2}^{i}\right) \rightarrow\left(x_{1}, x_{2}\right)$. After passing to a suitable subnet let $\left(\bar{x}_{1}, \bar{x}_{2}\right)=\lim t_{i}\left(x_{1}^{i}, x_{2}^{i}\right)$. Then

$$
\psi\left(\bar{x}_{1}\right)=\lim t_{i} \psi\left(x_{1}^{i}\right)=\lim t_{i} z_{1}^{i}=z_{1}=\lim t_{i} z_{2}^{i}=\lim t_{i} \psi\left(x_{2}^{i}\right)=\psi\left(\bar{x}_{2}\right),
$$

hence $\left(\bar{x}_{1}, \bar{x}_{2}\right) \in R_{\psi}$ and therefore $\left(\bar{x}_{1}, \bar{x}_{2}\right) \in Q_{\phi}=Q_{\phi}^{*}$. By 3.4b, it follows that $\left(x_{1}, x_{2}\right) \in$ $Q_{\phi}$. Consequently, $(\psi \times \psi)^{\leftarrow}\left[Q_{\theta}\right] \subseteq Q_{\phi}$ and as, clearly, $Q_{\phi} \subseteq(\psi \times \psi)^{-}\left[Q_{\theta}\right]$, it follows that $Q_{\phi}=(\psi \times \psi)^{-}\left[Q_{\theta}\right]$.

\section{Regional proximality of second order}

Let $\mathscr{X}$ be a ttg. It is not difficult to see that a pair $\left(x_{1}, x_{2}\right) \in X \times X$ is regionally proximal if we can find suitable pairs in the neighbourhood of $\left(x_{1}, x_{2}\right)$ such that 
after suitable $T$-translations they tend to a proximal pair. If we could find pairs in the neighbourhood of $\left(x_{1}, x_{2}\right)$ that after suitable $T$-translations tend to a regionally proximal pair, we could say that the pair $\left(x_{1}, x_{2}\right)$ is regionally regionally proximal. We call it regionally proximal of second order.

Let $\mathscr{X}$ be a ttg and let $A \subseteq X$. Then define

$$
D(A, \mathscr{X}):=\bigcup\left\{p * A \mid p \in S_{T}\right\}
$$

where $p * A$ is defined as

$$
p * A:=\bigcap\{p \circ V \mid A \subseteq V \text { and } V \text { open in } X\} .
$$

We remark that the $*$ defined in $\S 3$ is in complete agreement with this definition, after noting that $p * a:=p *\{a\}$.

(4.1) Remark. Let $\mathscr{X}$ be a ttg and let $A \subseteq X$. Then

(a) $D(A, \mathscr{Z})$ is T-invariant;

(b) $D(A, \mathscr{X})=D(t A, \mathscr{X})$ for every $t \in T$;

(c) if $A$ is closed then $D(A, \mathscr{X})=\bigcup\{D(\{a\}, \mathscr{X}) \mid a \in A\}$;

(d) if $A$ is closed then $D(A, \mathscr{Q})$ is closed.

Proof. (a) Let $x \in D(A, \mathscr{X})$ and let $p \in S_{T}$ be such that $x \in p * A$. Then $x \in p \circ V$ for every open $V$ in $X$ with $A \subseteq V$. Hence $t x \in t p \circ V$ for such $V$ and $t x \in t p * A \subseteq D(A, \mathscr{X})$.

(b) Note that $p \circ V=p t^{-1} \circ t V$ for every $V \subseteq X, p \in S_{T}$ and $t \in T$. As

$$
\{W \mid W \subseteq X \text { open, } t A \subseteq W\}=\{t V \mid V \subseteq X \text { open, } A \subseteq V\}
$$

for every $t \in T$, it follows that $p * A=p t^{-1} * t A$.

(c) Obviously, $D(\{a\}, \mathscr{X}) \subseteq D(A, \mathscr{X})$ for every $a \in A$.

Conversely, let $x \in D(A, \mathscr{Q})$ and let $p \in S_{T}$ be such that $x \in p * A$. Let $\alpha \in \mathscr{U}_{X}$ be an open index. Then there are $a_{1}, \ldots, a_{n}$ in $A$ such that

$$
V_{\alpha}:=\bigcup\left\{\alpha\left(a_{i}\right) \mid i \in\{1, \ldots, n\}\right\}
$$

is an open neighbourhood of $A$ (in $X$ ). So $x \in p \circ V_{\alpha}$, and as

$$
p \circ V_{\alpha}=\bigcup\left\{p \circ \alpha\left(a_{i}\right) \mid i \in\{1, \ldots, n\}\right\},
$$

we can find $a_{\alpha} \in\left\{a_{i} \mid i \in\{1, \ldots, n\}\right\}$ such that $x \in p \circ \alpha\left(a_{\alpha}\right)$. In this way we obtain a point $a_{\alpha}$ in $A$ for every open index $\alpha \in \mathcal{U}_{X}$. Let $a:=\lim \left\{a_{\alpha} \mid \alpha \in I\right\}$ for a suitable subnet $I \subseteq \mathcal{U}_{X}$. We shall prove that $x \in p *\{a\}$.

Let $V \subseteq X$ be open and let $\{a\} \subseteq V$. Then there are $\beta$ and $\gamma$ in $I$ such that $\beta(a) \subseteq V$ and $\gamma \circ \gamma \subseteq \beta$. Let $\delta \in I$ with $\delta \subseteq \gamma$ such that $a_{\delta} \in \gamma(a)$. Then

$$
x \in p \circ \delta\left(a_{\delta}\right) \text { and } \delta\left(a_{\delta}\right) \subseteq \gamma\left(a_{\delta}\right) \subseteq \gamma(\gamma(a)) \subseteq \beta(a) \text {, }
$$

so $x \in p \circ \delta\left(a_{\delta}\right) \subseteq p \circ \beta(a) \subseteq p \circ V$; hence $x \in p *\{a\}$. As $a \in \bar{A}=A$ it follows that

$$
D(A, \mathscr{X}) \subseteq \bigcup\{D(\{a\}, \mathscr{X}) \mid a \in A\} .
$$

(d) Let $\left\{x_{i}\right)_{i}$ be a convergent net in $D(A, \mathscr{X})$ and let $x=\lim x_{i}$. By (c), we may find nets $\left\{a_{i}\right\}_{i}$ and $\left\{p_{i}\right\}_{i}$ in $A$ and $S_{T}$ such that $x_{i} \in p_{i} *\left\{a_{i}\right\}$. Let $p=\lim p_{i}$ and $a=\lim a_{i}$ after passing to suitable subnets. We shall prove that $x \in p *\{a\}$.

Let $V \subseteq X$ be open with $\{a\} \subseteq V$. Then $\left\{a_{i}\right\} \subseteq V$ for all $i \geq i(V)$. Hence

$$
x_{i} \in p_{i} *\left\{a_{i}\right\} \subseteq p_{i} \circ V \quad \text { for all } i \geq i(V) .
$$


But then it follows that

$$
x=\lim x_{i} \in \lim _{2} x\left(p_{i} \circ V\right)=p \circ V .
$$

As $V$ was arbitrary, it follows that $x \in p *\{a\}$, hence $x \in D(A, \mathscr{X})$.

The proof of the following remark is straightforward and will be omitted.

(4.2) REMARK. For a ttg $\mathscr{X}, x \in X$ and $a \in X$ the following statements are equivalent:

(a) $x \in p * a$ for some $p \in S_{T}$, in other words $x \in D(\{a\}, \mathscr{X})$;

(b) for every $V_{a} \in \mathscr{V}_{a}$, and every $V_{x} \in \mathscr{V}_{x}$ there is a $t \in T$ such that $t V_{a} \cap V_{x} \neq \varnothing$;

(c) there is a net $\left\{a_{i}\right\}_{i}$ in $X$ with $a_{i} \rightarrow a$, and there are $t_{i}$ in $T$ with $x=\lim t_{i} a_{i}$;

(d) $a \in q * x$ for some $q \in S_{T}$, in other words $a \in D(\{x\}, \mathscr{X})$.

Note that this shows that for $p \in S_{T}$ and $x \in X$ we have $p * x \subseteq Q_{\mathscr{x}}[p x]$.

(4.3) ExAMPLEs. Let $\mathscr{X}$ be a ttg and let $\phi: \mathscr{X} \rightarrow \mathscr{Y}$ be a homomorphism of ttgs. Then

(a) $D\left(\Delta_{X}, \mathscr{Z} \times \mathscr{X}\right)=Q_{\mathscr{X}}$;

(b) $D\left(\Delta_{X}, \mathscr{R}_{\phi}\right)=Q_{\phi}$;

(c) $D\left(E_{\phi}, \mathscr{R}_{\phi}\right)=E_{\phi}$ and so $D\left(Q_{\phi}, \mathscr{R}_{\phi}\right) \subseteq E_{\phi}$;

(d) $D\left(Q_{\phi}^{\#}, \mathscr{R}_{\phi}\right)=Q_{\phi}$, hence $Q_{\phi}=Q_{\phi}^{*}$ implies $D\left(Q_{\phi}, \mathscr{R}_{\phi}\right)=Q_{\phi}$.

Proof. (a) Follows immediately from (b).

(b) Using 4.1c and 4.2 this follows easily from 3.13.

(c) Let $\theta: \mathscr{X} / E_{\phi} \rightarrow \phi[\mathscr{X}]$ be the maximal almost periodic factor of $\phi$ and let $\kappa: \mathscr{X} \rightarrow \mathscr{X} / E_{\phi}$ be the quotient map. Then it is easily seen that

$$
\kappa \times \kappa\left[D\left(E_{\phi}, \mathscr{R}_{\phi}\right)\right] \subseteq D\left(\Delta_{X / E_{\phi}}, \kappa \times \kappa\left[\mathscr{R}_{\phi}\right]\right) \subseteq Q_{\theta} .
$$

As $\theta$ is an almost periodic extension, $\kappa \times \kappa\left[D\left(E_{\phi}, \mathscr{R}_{\phi}\right)\right] \subseteq \Delta_{X / E_{\phi}}$; hence $D\left(E_{\phi}, \mathscr{R}_{\phi}\right) \subseteq$ $E_{\phi}$.

(d) Clearly, $Q_{\phi}=D\left(\Delta_{X}, \mathscr{R}_{\phi}\right) \subseteq D\left(Q_{\phi}^{*}, \mathscr{R}_{\phi}\right)$.

Conversely, as $Q_{\phi}^{*} \subseteq \operatorname{int}_{R_{\phi}}\left(\overline{T \alpha \cap R_{\phi}}\right)$ for every $\alpha \in \mathcal{U}_{X}$, we have

$$
p * Q_{\phi}^{*} \subseteq p \circ \text { int }_{R_{\phi}}\left(\overline{T \alpha \cap R_{\phi}}\right) \subseteq p \circ \overline{T \alpha \cap R_{\phi}} \subseteq \overline{T \alpha \cap R_{\phi}} \quad\left(\alpha \in U_{X}\right) .
$$

So $p * Q_{\phi}^{*} \subseteq Q_{\phi}$ and $D\left(Q_{\phi}^{*}, \mathscr{R}_{\phi}\right) \subseteq Q_{\phi}$.

The next theorem and its proof resemble 3.15 and 3.16 .

(4.4) THEOREM. Let $\phi: \mathscr{X} \rightarrow \mathscr{Y}$ be a homomorphism of ttgs. If for every $x_{1} \in X$ there is an $x \in X$ with $\overline{T x} \cap \overline{T x_{1}} \neq \varnothing$, such that $\phi(x)$ is an almost periodic point and $\phi$ is open in $x$, then $E_{\phi}=Q_{\phi}$ iff $D\left(Q_{\phi}, \mathscr{R}_{\phi}\right)=Q_{\phi}$.

Proof. If $E_{\phi}=Q_{\phi}$ then, by 4.3 , it follows that $D\left(Q_{\phi}, \mathscr{R}_{\phi}\right)=Q_{\phi}$.

Conversely, suppose that $D\left(Q_{\phi}, \mathscr{R}_{\phi}\right)=Q_{\phi}$. Let $\left(x_{1}, x_{2}\right) \in Q_{\phi}$ and $\left(x_{2}, x_{3}\right) \in Q_{\phi}$, and assume $\phi$ is open in $x_{1}$. We shall prove that $\left(x_{1}, x_{3}\right) \in Q_{\phi}$. Let $\left\{\left(x_{2}^{i}, x_{3}^{i}\right)\right\}_{i}$ and $\left\{t_{i}\right\}_{i}$ be nets in $R_{\phi}$ and $T$ such that

$$
\left(x_{2}^{i}, x_{3}^{i}\right) \rightarrow\left(x_{2}, x_{3}\right) \quad \text { and } \quad t_{i}\left(x_{2}^{i}, x_{3}^{i}\right) \rightarrow(w, w) \quad \text { for some } w \in X .
$$

As $\phi\left(x_{2}^{i}\right) \rightarrow \phi\left(x_{2}\right)=\phi\left(x_{1}\right)$ and as $\phi$ is open in $x_{1}$, there are $z_{i} \in \phi^{-} \phi\left(x_{2}^{i}\right)$ such that $z_{i} \rightarrow x_{1}$. Define $z=\lim t_{i} z_{i}$ (after passing to a suitable subnet). Then

$$
\left(z_{i}, x_{2}^{i}\right) \rightarrow\left(x_{1}, x_{2}\right) \text { and } t_{i}\left(z_{i}, x_{2}^{i}\right) \rightarrow(z, w) .
$$

As $\left(x_{1}, x_{2}\right) \in Q_{\phi}$ it follows that

$$
(z, w) \in p *\left(x_{1}, x_{2}\right) \subseteq D\left(\left\{\left(x_{1}, x_{2}\right)\right\}, \mathscr{R}_{\phi}\right) \subseteq D\left(Q_{\phi}, \mathscr{R}_{\phi}\right)=Q_{\phi},
$$


where $p=\lim t_{i} \in S_{T}$ (after passing to a suitable subnet). As $\left(z_{i}, x_{3}^{i}\right) \rightarrow\left(x_{1}, x_{3}\right)$ and $t_{i}\left(z_{i}, x_{3}^{i}\right) \rightarrow(z, w)$, it follows that

$$
\left(x_{1}, x_{3}\right) \in q *(z, w) \subseteq D\left(\{(z, w)\}, \mathscr{R}_{\phi}\right) \subseteq D\left(Q_{\phi}, \mathscr{R}_{\phi}\right)=Q_{\phi},
$$

where $q=\lim t_{i}^{-1} \in S_{T}$ (after passing to a suitable subnet). Now assume that $\phi$ is not open in $x_{1}$. By assumption, we may find $x \in X$ such that $\overline{T x} \cap \overline{T x_{1}} \neq \varnothing$ and $\phi$ is open in $x$, while $\phi(x) \in Y$ is an almost periodic point. For an almost periodic point $z \in \overline{T x} \cap \overline{T x_{1}}$ let $I$ and $K$ be minimal left ideals in $S_{T}$ such that $z=p x$ and $z=q x_{1}$ for some $p \in I$ and some $q \in K$. Let $v \in J_{\phi(x)}(I)$. Then $v x=v p^{-1} q x_{1}$, and

$$
\left(v x, v p^{-1} q x_{2}\right)=v p^{-1} q\left(x_{1}, x_{2}\right) \in Q_{\phi}
$$

and

$$
\left(v p^{-1} q x_{2}, v p^{-1} q x_{3}\right) \in Q_{\phi} .
$$

As $(x, v x) \in P_{\phi}$, we have $\left(x, v p^{-1} q x_{2}\right) \in Q_{\phi} \circ P_{\phi}$ and it is easily seen that $Q_{\phi} \circ P_{\phi} \subseteq$ $D\left(Q_{\phi}, \mathscr{R}_{\phi}\right)=Q_{\phi}$. By the above, $\left(x, v p^{-1} q x_{3}\right) \in Q_{\phi}$ and so

$$
v p^{-1} q\left(x_{1}, x_{3}\right)=\left(v p^{-1} q x_{1}, v p^{-1} q x_{3}\right)=\left(v x, v p^{-1} q x_{3}\right)=v\left(x, v p^{-1} q x_{3}\right) \in Q_{\phi} .
$$

But then

$$
\left(x_{1}, x_{3}\right) \in D\left(\left\{\left(v p^{-1} q x_{1}, v p^{-1} q x_{3}\right)\right\}, \mathscr{R}_{\phi}\right) \subseteq D\left(Q_{\phi}, \mathscr{R}_{\phi}\right)=Q_{\phi},
$$

which shows the transitivity of $Q_{\phi}$.

(4.5) COROLlaRY. Let $\phi: \mathscr{X} \rightarrow \mathscr{Y}$ be a homomorphism of ttgs.

(a) If $\phi$ is open then $E_{\phi}=Q_{\phi}$ iff $D\left(Q_{\phi}, \mathscr{R}_{\phi}\right)=Q_{\phi}$. In particular, for every ttg $\mathscr{X}$ we have $E_{\mathscr{X}}=Q_{\mathscr{X}}$ iff $D\left(Q_{\mathscr{X}}, \mathscr{X} \times \mathscr{X}\right)=Q_{\mathscr{X}}$.

(b) If $\mathscr{X}$ is a metric ergodic ttg and if $\mathscr{Y}$ is minimal, then $E_{\phi}=Q_{\phi}$ iff $D\left(Q_{\phi}, \mathscr{R}_{\phi}\right)=Q_{\phi}$.

Proof. (a) This follows immediately from the first part of the proof of 4.4 .

(b) If $X$ is metric, there is a residual set of points in which $\phi$ is open, also there is a residual set of transitive points. As $\mathscr{Y}$ is mimimal, the assumptions of 4.4 are satisfied.

\section{REFERENCES}

[1] J. Auslander \& J. C. S. P. van der Woude. Maximally highly proximal generators of minimal flows. Ergod. Th. \& Dynam. Sys. 1 (1981), 389-412.

[2] I. U. Bronstein. Stable and equicontinuous extensions of minimal sets. Math. Issled. 8 (1973), 3-11. (Russian).

[3] I. U. Bronstein. Extensions of Minimal Transformation Groups. Sijthoff \& Noordhoff: Alphen aan den Rijn, 1979. (Russian edition: 1975).

[4] R. Ellis. Group-like extensions of minimal sets. Trans. Amer. Math. Soc. 127 (1967), 125-135.

[5] R. Ellis. Lectures on Topological Dynamics. Benjamin: New York, 1969.

[6] R. Ellis. The Veech structure theorem. Trans. Amer. Math. Soc. 186 (1973), 203-218.

[7] R. Ellis, S. Glasner \& L. Shapiro. Proximal isometric ( $\mathscr{P} \mathscr{S}_{-}$) flows. Adv. in Math. 17 (1975), 213-260.

[8] H. Furstenberg. The structure of distal flows. Amer. J. Math. 85 (1963), 477-515.

[9] S. Glasner. Relatively invariant measures. Pacific J. Math. 58 (1975), 393-410.

[10] S. Glasner. Proximal Flows. Lecture Notes in Math. 517, Springer Verlag: New York 1976.

[11] D. C. McMahon. Relativized weak disjointness and relatively invariant measures. Trans. Amer. Math. Soc. 236 (1978), 225-237.

[12] D. C. McMahon \& T. S. Wu. Notes on topological dynamics V: equicontinuous structure relations of minimal transformation groups. Bull. Inst. Math. Acad. Sinica. 8 (1980), 283-294. 
[13] E. Michael. Topologies on spaces of subsets. Trans. Amer. Math. Soc. 71 (1951), 152-182.

[14] R. Peleg. Weak disjointness of transformation groups. Proc. Amer. Math. Soc. 33 (1972), 165-170.

[15] W. A. Veech. Topological dynamics. Bull. Amer. Math. Soc. 83 (1977), 775-830.

[16] J. C. S. P. van der Woude. Weakly mixing remarks. In Rep. ZN 99, Mathematisch Centrum: Amsterdam, 1980. 